\title{
Synchronization and chaos control in a periodically forced quasi-geostrophic two-layer model of baroclinic instability
}

\author{
F. J. R. Eccles ${ }^{1}$, P. L. Read $^{1}$, and T. W. N. Haine ${ }^{2}$ \\ ${ }^{1}$ Department of Physics, Clarendon Laboratory, Parks Road, Oxford, OX1 3PU, UK \\ ${ }^{2}$ Department of Earth and Planetary Sciences, Johns Hopkins University, Baltimore, Maryland 21218, USA \\ Received: 4 July 2005 - Revised: 20 December 2005 - Accepted: 2 January 2006 - Published: 22 February 2006
}

\begin{abstract}
.
Cyclic forcing on many timescales is believed to have a significant effect on various quasi-periodic, geophysical phenomena such as El Niño, the Quasi-Biennial Oscillation, and glacial cycles. This variability has been investigated by numerous previous workers, in models ranging from simple energy balance constructions to full general circulation models. We present a numerical study in which periodic forcing is applied to a highly idealised, two-layer, quasi-geostrophic model on a $\beta$-plane. The bifurcation structure and (unforced) behaviour of this particular model has been extensively examined by Lovegrove et al. (2001) and Lovegrove et al. (2002). We identify from their work three distinct regimes on which we perform our investigations: a steady, travelling wave regime, a quasi-periodic, modulated wave regime and a chaotic regime. In the travelling wave regime a nonlinear resonance is found. In the periodic regime, Arnol'd tongues, frequency locking and a Devil's staircase is seen for small amplitudes of forcing. As the forcing is increased the Arnol'd tongues undergo a period doubling route to chaos, and for larger forcings still, the parameter space we explored is dominated by either period 1 behaviour or chaotic behaviour. In the chaotic regime we extract unstable periodic orbits (UPOs) and add the periodic forcing at periods corresponding to integer multiples of the UPO periods. We find regions of synchronization, similar to Arnol'd tongue behaviour but more skewed and centred approximately on these periods. The regions where chaos suppression took place are smaller than the synchronization regions, and are contained within them.
\end{abstract}

Correspondence to: P. L. Read

(p.read1@physics.ox.ac.uk)

\section{Introduction}

Examples of cyclic climatic phenomena are widespread, and there has been much attention given to reproducing this behaviour in climate models of greatly varying degrees of complexity. Interactions between various external forcings (due to astronomical modulations of the solar input) and possible intrinsic climatic oscillations form a significant subset of these investigations.

The range of timescales for these different forcings are vast. Models of the glacial cycles cover the last few hundred thousand years (e.g. Imbrie and Imbrie, 1980; Ghil and Le Treut, 1981; Saltzman et al., 1982; Nicolis, 1984). On much shorter timescales many authors have attempted to reproduce the Quasi-Biennial Oscillation (see Baldwin et al., 2001, for example for a review), some including forcing with an annual cycle (Geller et al., 1997, for example). The annual cycle is also believed to play a role in the El Niño Southern Oscillation (ENSO), though its precise influence is still under debate (e.g. Jin et al., 1996). Yet another example of the periodic forcing is seen in the recent work on interactions between oceanic tropical instability waves and annual (Vialard et al., 2003) and sub-annual (Allen et al., 1995; Benestad et al., 2001) wind forcings.

Another source of atmospheric variability, perhaps more closely related to the present work, is the the North Atlantic Oscillation, or NAO (e.g. Wallace, 2000). This has fluctuations on multiple timescales though the relative contributions of the components may not be constant in time (Higuchi et al., 1999). Possible causes of the 2-3 year variability, for instance, include annual modulation of coupled atmosphericsurface feedbacks, or subharmonics of the annual cycle generated by mid-latitude non-linearities (Stephenson et al., 2000). Wallace (2000) showed that NAO indices, measures of the strength of the oscillation, are effectively the same as those developed by Lorenz (1951) for the zonal index cycle (e.g. Rossby, 1939; Namias, 1950). This views the variability from an annular mode paradigm.

Published by Copernicus GmbH on behalf of the European Geosciences Union and the American Geophysical Union. 
A similar effect of annular modes is seen in the amplitude modulations or "vacillations" of rotating annulus experiments such as those of Hart (1972), Hide and Mason (1975), Pfeffer et al. (1980) or Früh and Read (1997). The transport of heat and angular momentum effected by "vacillating" waves in the annulus (which have periodic amplitude modulation) varies with a similar period to the changing shape and intensity of the wave, thus producing a periodic "zonal index" variation. Southern Hemisphere baroclinic waves have also been seen to vary in a similar way with timescales of 20-60 days (e.g. Randel and Stanford, 1985a).

In this paper we present experiments with an idealised two-layer quasi-geostrophic model of baroclinic instability (Lovegrove et al., 2001, 2002, hereafter LMR1 and LMR2, respectively. We shall use LMR to refer jointly to both papers and the reader is referred to these papers for details.) This model is arguably the simplest geophysical flow representing the quasi-geostrophic flow within the ocean or atmosphere. It also models certain flows seen in annulus experiments mentioned above. The model is formulated in Cartesian geometry and models the interaction of a single mixed baroclinic-barotropic wave mode with a background zonal flow.

The previous investigations of LMR with this model found a rich sequence of bifurcations including pitchfork, Hopf and secondary Hopf bifurcations, and period doubling cascades leading to regimes corresponding to a stationary wave, a travelling wave, an amplitude modulated wave and chaotic regimes, respectively. LMR studied the bifurcation structure as three parameters were varied, namely the dissipation parameter, $r$, the planetary vorticity gradient, $\beta$ and the Froude number $F$. They thus unified various previous multiple-scale studies which had considered only limited choices of these three parameters. (For a summary see Table 1 in LMR1.) In particular they note that, for small values of $\beta$ and $r$, the spectral amplitude equations reproduce the behaviour associated with the complex Lorenz equations (Fowler et al., 1982), while for an $f$-plane model (i.e. for $\beta=0$ ) the equations reduce to the Lorenz equations with real coefficients (Lorenz, 1963).

In the present study we add periodic forcing to the model and examine the ensuing behaviour. We consider three distinct regimes which were found by LMR:

- The steady wave (limit cycle) regime.

- The amplitude vacillation (quasi-periodic modulated wave) regime.

- A chaotic regime with large Froude number.

Hart has also considered adding a periodic modulation to a two-layer model on both an $f$-plane (Hart, 1989a) and a $\beta$-plane (Hart, 1989b). In both cases he applied a periodic modulation in a similar way: the parameter representing the mean flow correction was varied, but only in the equation for the evolution of the baroclinic term. In contrast we vary the baroclinic velocity directly as, arguably, a physical way of introducing an oscillatory forcing in the background thermal structure, given that such a forcing may arise in the atmosphere due to differential solar heating, or in the laboratory by changing the thermal boundary conditions of a differentially heated annulus. The heat input into the system oscillates which in turn affects the baroclinic part of the flow via the thermal wind equation. Hart (1989b), in his $\beta$-plane study, only considered forcing at timescales which were very different in magnitude from the natural internal frequency of the system. We, however, choose to concentrate on timescales of the same order as frequencies identified from the unforced system.

Pedlosky and Thomson (2003) considered adding an oscillatory perturbation to a similar Phillips-type model to the one considered here, approaching the problem from a different perspective. They introduced an oscillatory shear flow in the neighbourhood of the classical steady-shear threshold for marginal stability. They found the perturbations changed the stability of the system, leading to new sources of growing disturbances which gave rise to new oscillatory states and new regions of instability and chaos. In the weakly nonlinear regime they show that the addition of periodic forcing on timescales comparable with the linear growth of baroclinic instability often leads to the development of chaotic oscillations for strong enough forcing. They also found that periodic forcing of sub-critical (baroclinically stable) flows could also lead to the development of oscillatory and chaotic instabilities if the periodic forcing allowed the shear to enter the linearly unstable regime even briefly during a cycle.

Our study covers more strongly supercritical states than considered by Pedlosky and Thomson (2003), albeit given the same wavenumber truncations. We find some broadly similar features to Pedlosky and Thomson (2003) with the appearance of an oscillatory state in previously steady wave regimes. Moreover we also find a nonlinear resonance. In the periodic regime we also see a range of aperiodic and even chaotic behaviour. In particular Arnol'd tongues, frequency locking and a Devil's staircase is seen for small amplitudes of forcing. As the forcing is increased the Arnol'd tongues undergo a period doubling route to chaos, and for larger forcings still, the parameter space we explored is dominated by either period 1 behaviour or chaotic behaviour. A summary of the findings, to be presented in more detail below, can be found in Fig. 1.

In Sect. 2 we briefly present the model and the methodologies used to identify the different states of the system, concentrating on the degree of synchronization and chaos. The detailed results of the study are in Sect. 3, with a summary and conclusion in Sect. 4. 


\section{Periodic Regime}
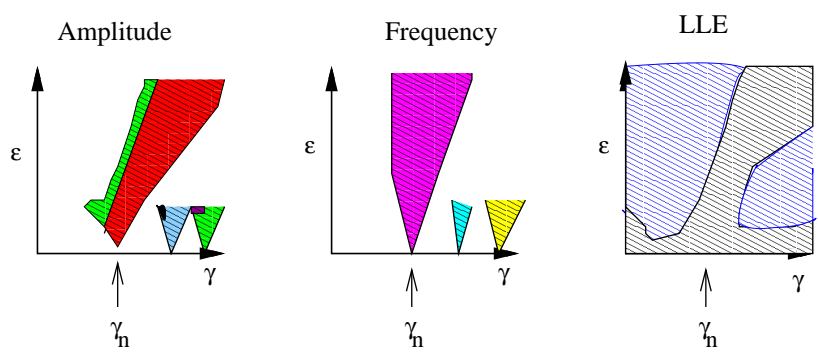

Chaotic Regime

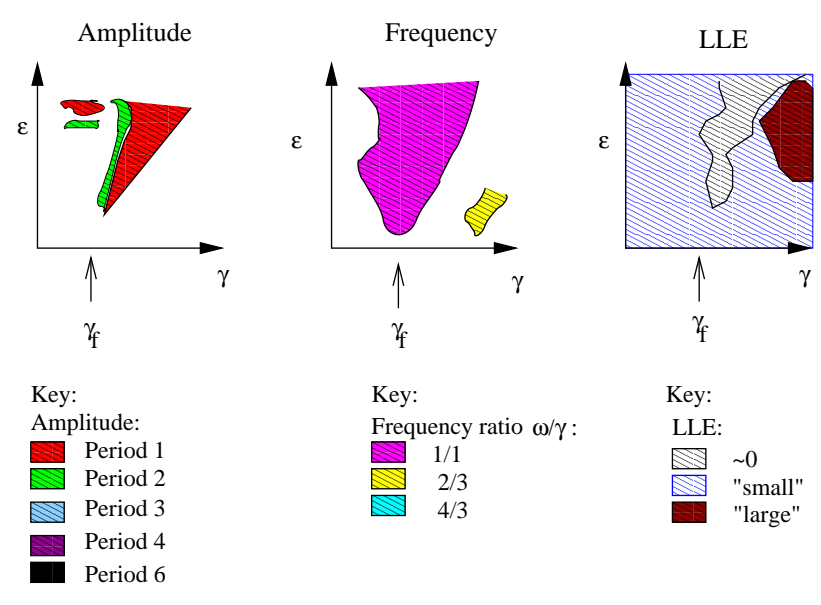

Fig. 1. Summary of the periodic and chaotic regimes. $\gamma_{n}$ (the natural frequency) largely performs a similar function to $\gamma_{f}$ (the frequency of the principal unstable periodic orbit); the Arnol'd tongue tends to $\gamma_{n}$ or $\gamma_{f}$ as $\epsilon \rightarrow 0$. In the periodic regime classic Arnol'd tongues are seen for small amplitudes of forcing. Period doubling and chaotic behaviour is visible for larger forcing amplitudes, with only the main tongue, now skewed, remaining for the largest amplitudes. A similar pattern in seen in the largest Lyapunov exponent (LLE). In the chaotic regime chaos is suppressed for particular values of $\gamma$, close to multiples of $\gamma_{f}$, and the chaos suppressed region increases with increasing $\epsilon$. This tongue however has regions where the chaos returns, and also not all points that are stable have stability for all initial conditions. The regions of synchronization defined by frequency locking are more extensive than those defined by the LLE. For further explanation see text.

\section{Methods}

\subsection{The model formulation}

The model to be considered is shown schematically in Fig. 2 and is the classic "Phillips-Pedlosky" two-layer model (Phillips, 1954; Pedlosky, 1970, 1971, 1972). The two superposed fluids are confined to a rotating, rectangular, zonally periodic channel of height $D$ and width $L$. We use a Cartesian co-ordinate system, $x_{*}, y_{*}$ and $z_{*}$ to describe distances along the channel, across the channel and vertically,

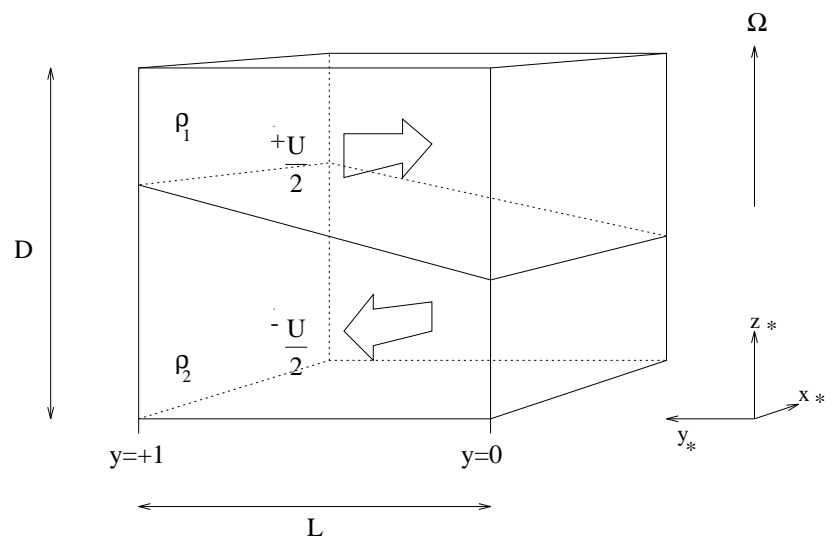

Fig. 2. The two-layer model considered in this study: comprising two immiscible fluids of densities $\rho_{1}$ and $\rho_{2}$ with $\rho_{1}<\rho_{2}$. The fluids are in relative motion, with velocities of $+U / 2$ and $-U / 2$, respectively. The channel has height $D$, width $L$ and is rotating at a constant angular velocity $\Omega$ (from Lovegrove, 1997).

respectively, with periodic boundary conditions in $x_{*}$ (starred variables refer to dimensional variables). The corresponding velocities are $u_{n *}, v_{n *}$ and $w_{n *}$, where the subscript $n=1(2)$ refers to the upper (lower) layer. The densities of the upper and lower layer are $\rho_{1}$ and $\rho_{2}$, with $\rho_{2}>\rho_{1}$. The analysis in LMR1 is carried out in a reference frame that is moving with the speed, $\left(u_{1 *}+u_{2 *}\right) / 2$, of the mean flow. In this frame, both the upper and lower layers appear to be in uniform motion along the channel, with respective velocities of $+U / 2$ and $-U / 2$; in non-dimensional units these become $+1 / 2$ and $-1 / 2$. In the absence of motion the fluid layers have equal depths of $D / 2$. The fluid is confined vertically between two horizontal planes representing a base and a lid. The kinematic viscosity within both layers is assumed to be equal. The principal dissipation is due to Ekman layers at the top and bottom of the model. There is also an internal dissipation term parameterised by potential vorticity diffusion. For further details see LMR1.

In LMR1 the barotropic and baroclinic streamfunctions $\psi_{s, d}$ are given by

$$
\begin{aligned}
& \psi_{s}=\left(\psi_{1}+\psi_{2}\right) / 2, \\
& \psi_{d}=\left(\psi_{1}-\psi_{2}\right) / 2 .
\end{aligned}
$$

where

$u_{q}=-\frac{\partial \psi_{q}}{\partial y} \quad$ and $\quad v_{q}=\frac{\partial \psi_{q}}{\partial x}$,

and $q=1$ or 2. $\psi_{s, d}$ are projected onto a series of Fourier modes:

$$
\psi_{s, d}=\underbrace{\sum_{m=1}^{M} X_{m}^{s, d} \cos l_{m} y}_{\text {mean flow correction }}+
$$

Nonlin. Processes Geophys., 13, 23-39, 2006 


$$
\underbrace{\sum_{\substack{n=-N \\ n \neq 0}}^{N} \sum_{m=1}^{M} W_{n m}^{s, d} \exp \left[i k_{n} x\right] \sin l_{m} y}_{\text {wave term }}-\underbrace{U_{s, d y}}_{\text {background }},
$$

where

$W_{-m n}^{s, d}=\left(W_{m n}^{s, d}\right)^{*} \quad$ and $\quad k_{-n}=-k_{n}$.

Subscripts $s$ and $d$ refer to the barotropic and baroclinic terms, respectively, and $U_{s, d}$ is the background flow; $U_{d}=1 / 2$ and $U_{s}=0$. The terms are truncated at one term in each direction, i.e. $M=N=1$. The resulting spectral amplitude equations for this 2-layer model (given as Eq. 11 in LMR2) are

$$
\begin{aligned}
& \dot{A_{s}}=-\Delta_{s} A_{s}+\beta_{s} B_{s}-\left(v_{s}+\gamma_{s} X_{d}\right) B_{d}, \\
& \dot{B_{s}}=-\Delta_{s} B_{s}-\beta_{s} A_{s}+\left(v_{s}+\gamma_{s} X_{d}\right) A_{d}, \\
& \dot{A_{d}}=-\Delta_{d} A_{d}+\beta_{d} B_{d}-\left(v_{d}+\gamma_{d} X_{d}\right) B_{s}, \\
& \dot{B_{d}}=-\Delta_{d} B_{d}-\beta_{d} A_{d}+\left(v_{d}+\gamma_{d} X_{d}\right) A_{s}, \\
& \dot{X_{d}}=-\bar{\Delta} X_{d}+\bar{\gamma}\left[A_{s} B_{d}-B_{s} A_{d}\right],
\end{aligned}
$$

where the dot represents differentiation with respect to time, $A_{s, d}=\operatorname{Re}\left(W_{11}^{s, d}\right)$, and $B_{s, d}=-\operatorname{Im}\left(W_{11}^{s, d}\right)$. The coefficients are somewhat unwieldy and are thus omitted here for brevity but are given in the appendix of LMR2. The equation for $\dot{X}_{s}$ is omitted as it describes only the simple exponential decay of $X_{s}$. (Note this occurs because we chose to have equal depths of the two layers of the model. An unequal depth model would lead to a 6 dimensional system, with non-zero $X_{s}$.)

To add the periodic forcing we vary the baroclinic velocity, $U_{d}$, directly via

$U_{d} \rightarrow U_{d}+\epsilon \sin (\gamma t)$

where $\epsilon$ is the amplitude of the oscillation and $\gamma$ the angular frequency. Modulating $U_{d}$ modulates the parameters $v_{s}$ and $v_{d}$ in Eq. (5).

In practice Eq. (5), including the modulation of $U_{d}$ given by Eq. (6), were integrated using Matlab's variable order Runge-Kutta solver.

\subsection{Diagnostics}

The solutions of Eq. (5) are analysed using four main techniques:

- bifurcation diagrams of the amplitude behaviour of $X_{d}$ which show visually and qualitatively the changing flow states

- Lyapunov exponents which give an indication as to whether the system is chaotic

- phase dynamics of $X_{d}$ which show whether there is synchronization present
- unstable periodic orbits (UPOs) (for the chaotic regime) which show if there are "natural frequencies" present in the system

These are discussed in turn below.

\subsubsection{Bifurcation diagrams}

To examine qualitatively how the flow state changes as a parameter varies, bifurcation diagrams can be conveniently constructed in the following way. The model runs with one set of parameters for 50000 (200 000) timesteps for the regular (chaotic) regime and the first 10000 (100 000) timesteps, representing the transient behaviours are discarded. The mean flow correction term $\left(X_{d}\right)$ is recorded and the successive minima and maxima of this variable are extracted, denoted $X_{\min }$ and $X_{\max }$.

To examine the bifurcation structure as a function of $\gamma$, for example, we increment $\gamma$ and rerun the model again for 50000 (regular regime) or 200000 (chaotic regime) timesteps. The initial conditions are the final values of the variables of the previous run. $X_{\min }(\gamma)$ and $X_{\max }(\gamma)$ then are plotted as a function of $\gamma$.

\subsubsection{Lyapunov exponents}

The spectrum of Lyapunov exponents indicate whether or not the system is chaotic. We used the method of Wolf et al. (1985) for numerically estimating the value of the largest Lyapunov exponent (LLE) within the model phase space. An initial-value numerical integration is performed from which the transients are allowed to decay. Two neighbouring points are then tracked in the phase space for a predetermined length of time during which the average rate of expansion is calculated. When the points become further apart than some specified amount two new neighbouring points are selected and the process repeated until the estimates of the exponents converge. ${ }^{1}$ The Lyapunov exponent, $\lambda$, is given by

$\lambda=\lim _{t \rightarrow \infty} \frac{1}{t} \log _{2} \frac{p(t)}{p(0)}$,

where $t$ is time and $p(t)$ is the length of the first ellipsoidal principal axis at time $t$. Time is scaled as $t=L t_{*} / U$ (see LMR1); we call one unit of time $\sigma$, and the LLEs quoted for the model results are all in terms of ${ }^{2}$ bits $/ \sigma$.

\subsubsection{Phase dynamics}

Another way of measuring interaction and synchronization is to investigate the phase relationship between the applied forcing, $\epsilon \sin (\gamma t)$ and some observable of the system. First

\footnotetext{
${ }^{1}$ This method actually yields the spectrum of Lyapunov exponents. However we only examined the leading exponent to diagnose whether or not the system is chaotic

${ }^{2} \mathrm{~A}$ "bit" is a power of 2 for amplification of amplitude; note $\lambda$ is defined in terms of $\log _{2}$.
} 
we must define what we mean by the phase in this system. For this we follow the ideas outlined by e.g. Pikovsky et al. (2003), using the analytic signal concept, originally introduced by Gabor (1946).

For the observable in our model we chose $X_{d}$, the mean flow correction, since this represents the nonlinear feedbacks on the mean flow, and is also used for the bifurcation diagrams above. Firstly the time mean is subtracted from $X_{d}(t)$ to give a timeseries $X(t)^{\prime}$ (e.g. Pikovsky et al., 2003). Then we calculate $\zeta(t)=X(t)^{\prime}+i X_{H}(t)^{\prime}$, where the subscript $H$ indicates the Hilbert transform of the signal. From $X^{\prime}$ and $X_{H}^{\prime}$, the real and imaginary parts of $\zeta$, we construct the phase,

$\phi_{x}(t)=\arctan \left[\frac{X_{H}(t)^{\prime}}{X(t)^{\prime}}\right]$.

We then calculate the time dependent phase difference,

$\Delta \phi(t)=\phi_{x}(t)-\gamma t$,

and look for stationary and non-stationary values of $\Delta \phi(t)$ to indicate phase synchronization. It should be noted that the analytic signal provides only an estimate of the true phase, which should increase linearly with time. Obviously the phase difference as defined using this concept may alter over one period of the forcing as the phase of one oscillator speeds up/slows down relative to the other. Also there may be partial locking, such that the phase difference largely remains stationary but occasionally performs phase jumps. To identify this type of behaviour and remove the variation in $\Delta \phi(t)$ over a period we calculate

$\Delta \phi_{M}=\Delta \phi \bmod 2 \pi$,

and then further subsample this at intervals of the period. In effect, we take a Poincaré section of $\Delta \phi(t)$ on the interval $(-\pi, \pi)$. We then plot a histogram (or probability density function, PDF) of the occurrence of $\Delta \phi_{M}$. Thus phase locking will appear as peaks in the histogram, and 1:1 locking as plateaux in $\Delta \phi(t)$.

\subsubsection{Terminology}

It is necessary to introduce a terminology to describe the amplitude and the phase behaviour. We follow Kociuba and Heckenburg's (2002) study of the complex Lorenz equations. We consider a system forced by a signal such as the top waveform in Fig. 3. To describe the amplitude periodicity we refer to the number of successive response peaks needed for recurrence - hence "period 1", "period 2", and so on. So, for example, waveforms a and b in Fig. 3 have period 1 amplitude periodicity (they reach the same amplitude after one peak) and waveforms $\mathrm{c}$ and $\mathrm{d}$ have period 2. To describe the frequency response we calculate the average phase velocity, $\omega=<d \phi_{x} / d t>\equiv \dot{\phi}$, where the $<>$ denote averaging over the run. We then use this definition to discuss frequency locking ratios of the forcing frequency, $\gamma$. Locking occurs when

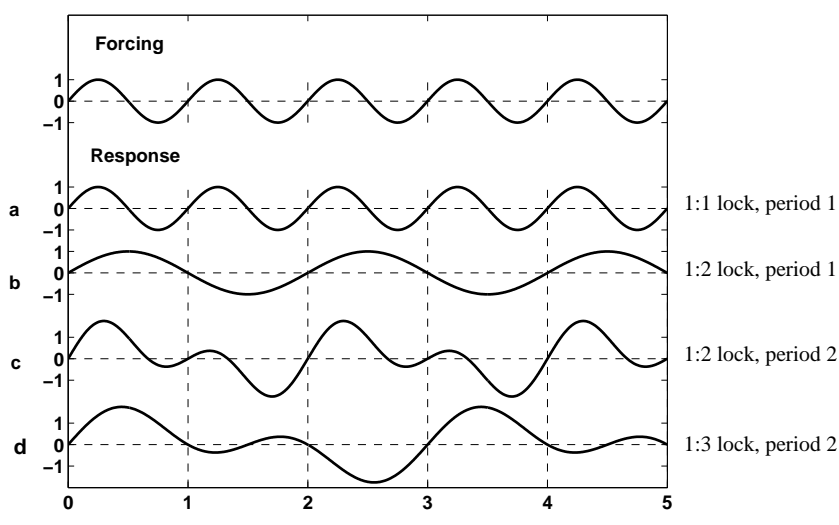

Fig. 3. A schematic to introduce the terminology of different locking ratios and behaviour of the period. The first waveform is the forcing, and all four waveforms represent possible responses. See text for further explanation.

$\omega / \gamma \approx n / m$ where $n$ and $m$ are integers. So, waveform a is locked in a $1 / 1$ ratio, waveforms $\mathrm{b}$ and $\mathrm{c}$ have ratio $1 / 2$, and waveform $\mathrm{d}$ has ratio $1 / 3$.

We acknowledge that this is not the only possible terminology, nor in some cases, will it be the most obvious one. However it is used throughout for consistency.

\subsubsection{Unstable periodic orbits}

In the chaotic regime certain forcing frequencies and amplitudes may suppress the chaos. For example, previous workers have suppressed chaos using perturbation frequencies that are rational multiples of periodic drive frequencies that initiated the chaos (e.g. Braimin and Goldhirsch, 1991; Fronzoni et al., 1991; Vohra et al., 1995), or frequencies corresponding to peaks in the power spectrum of the undriven system (e.g. Sätherblom, 1997; Ding et al., 1994).

Mirius and Sprott (1999) suggest that these predictors are not always reliable, but that the optimum perturbation frequencies more typically correspond to rational multiples of the frequencies of the unstable periodic orbits (UPOs) embedded in the chaotic attractor. The UPOs can, in principle, be extracted from the time series of any state-space variable and hence no specific model for the system dynamics is required to predict optimum perturbation frequencies. Mirius and Sprott (1999) apply their method to a variety of systems including the Lorenz equations, and we follow them here (see also Lathrop and Kostelich, 1989). The first step is to identify the UPOs present in the attractor. Firstly the attractor is reconstructed from the time series of $X_{d}(t)$, using time delays for simplicity, and then we measure the number of steps it takes for the trajectory to return to within a distance $\delta$ of any given point. If this number of steps is $m$ it is called an $m$ recurrence point. When the number of such points are plotted in a histogram, as a function of $m$, the data tend to cluster at regular intervals, separated by a constant $\Delta m$. 


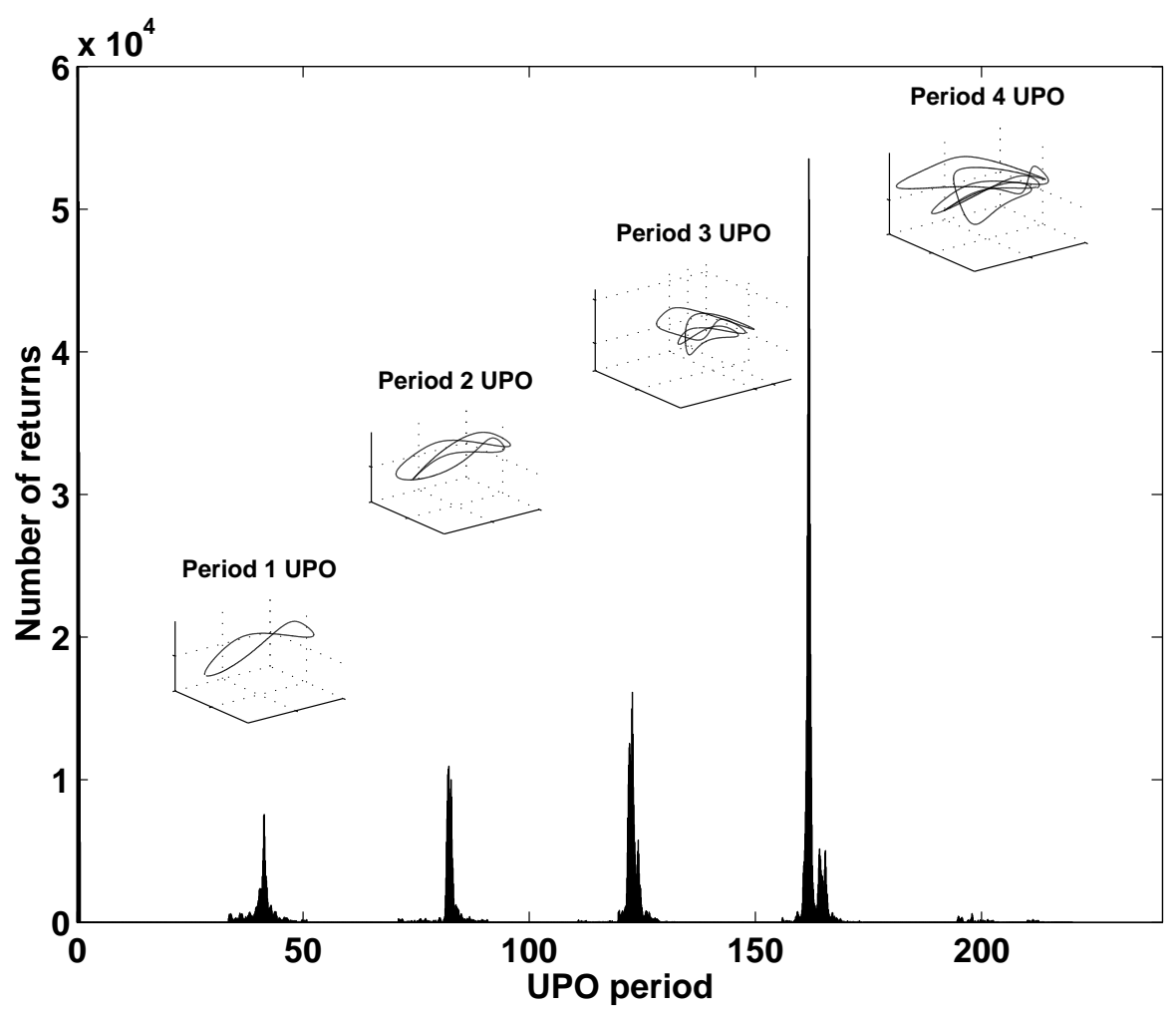

Fig. 4. Histogram of recurrence points for $\delta=0.0008$. Inserts show examples of individual UPOs at period 1, 2, 3 and 4 which give rise to the peaks in the histogram. For parameters see LMR.

Table 1. Table of parameters used for each regime. All experiments had Rossby number of 0.05 . This is obviously small although finite, and is consistent with the conventional quasi-geostrophic approximation.

\begin{tabular}{lccc}
\hline Regime & $r$ & $F$ & $\beta$ \\
\hline Travelling Wave & 0.02 & 5.5 & 0.1 \\
Amplitude Vacillation & 0.02 & 5.6 & 0.1 \\
Chaotic & 0.08 & 11.0 & 0.1 \\
\hline
\end{tabular}

For example, the histogram of number of returns is in Fig. 4 for our system of interest. Here, $\Delta m=40.45 \pm 0.17$, and there exist individual UPOs at period 1, 2, 3 and 4 . We call the period $\tau_{f}=40.45$, the fundamental UPO period, and the associated angular frequency $\gamma_{f} \equiv 2 \pi / \tau_{f} \approx 0.1553$.

\section{Results}

We now present the results of our numerical experiments investigating the behaviour of Eq. (5) when a periodic forcing term is added. LMR demonstrated that even when unforced, the model generates a wide variety of behaviour depending on the regime of parameter space chosen for investigation. It was unfeasible to perform an exhaustive study on the effect of adding a periodic forcing to this model. The results presented are, therefore, necessarily selective, and are chosen to demonstrate examples of the rich structure that the model can exhibit. They also provide examples of synchronization phenomena in a model with geophysical relevance.

We concentrate on just three points in the $(F, r, \beta)$ parameter space of LMR in the three principal regimes: (i) the travelling steady wave regime, (ii) an amplitude vacillation regime and (iii) a chaotic regime, explored in Sects. 3.1, 3.2 and 3.3, respectively. We concentrate on the latter two. The parameters for each regime were chosen from the work of LMR and can be seen in Table 1. In the steady regime the mean flow correction term, $X_{d}$, is constant in time and nonzero, in the amplitude vacillation regime it varies periodically, and in the chaotic regime a range of amplitudes and frequencies are seen. We present the results of several experiments in which either the amplitude, $\epsilon$, or the frequency, $\gamma$, of the forcing is fixed and the other is varied gradually in steps.

\subsection{The travelling wave regime}

In the travelling wave regime we do not expect to see synchronization since there is no "natural frequency" of 
the system. Rather, we observe how the oscillatory forcing changes this previously oscillation-free state.

Figure 5 shows a plot of the amplitude envelope of the mean flow correction for various values of $\epsilon$, plotted as functions of $\gamma$, i.e. $X_{\min }$ and $X_{\max }$ are shown in the same colour for a particular value of $\epsilon$. Recall that, without forcing there is no "natural" frequency of the system - there are no oscillations, hence the amplitude of the mean flow correction for $\epsilon=0$ is a constant in Fig. 5 ( $X_{\min }=X_{\max }$, shown in light blue). There appears to be a (nonlinear) resonance around $\gamma=0.008-0.009$, in which the modulation amplitude increases. Is this resonance perhaps showing the existence of a UPO around this frequency? LMR2 studied the period of the unstable torus born in a subcritical torus bifurcation for this set of parameters as $F$ was decreased (see their Fig. 3). They found the period increased as $F$ was decreased, but reached a period of 200-300 before disappearing in the saddle-node bifurcation at around $F=5.6$. We are below this at $F=5.5$, where the torus has, according to LMR1, disappeared. Also we have a larger period of around 700 (for $\gamma=0.009$, see Fig. 5), but speculate that the resonance may be related to this torus. The near-resonant perturbation of a system close to a bifurcation point can sometimes suppress or induce the bifurcation to chaos i.e. move the bifurcation point (e.g Lima and Pettini, 1990; Vohra et al., 1995). We speculate that the same may be happening here and thus the torus like behaviour may be found for larger values of $F$ in the forced system than in the unforced one. Note we are considering only the mean flow correction term. The wave terms have an additional oscillatory term, and hence a limit cycle in the mean flow correction term, $X_{d}$, implies a torus in the full system.

The oscillations visible for $\epsilon=0.2$ (shown in green), which appear to have a variable amplitude (around $\gamma=0.014$ ), are in fact long lived transients (longer than our rejected first 10000 data points ${ }^{3}$ ), but indicate a period doubling is about to take place. Certainly for $\epsilon=0.5$ (not shown), clear period doublings become evident, followed then by inverse period doublings as $\gamma$ increases. For values of $\epsilon \gtrsim 0.3$ chaotic behaviour identified by an LLE $>0$ was found for some values of $\gamma$.

\subsection{Periodic amplitude vacillation}

In the studies of LMR the steady travelling wave underwent a Hopf bifurcation at larger $F$ to a regime in which the amplitude of the wave and mean flow correction terms varied periodically - an amplitude vacillation regime. The principal frequency of oscillation, $\gamma_{n}=0.0995$, is the "natural" frequency of the system. We now explore this regime by ramping $\gamma$ with $\epsilon$ fixed, and vice versa. The bifurcation diagrams are plotted as functions of the varying parameter, and in all

\footnotetext{
${ }^{3}$ The question will often arise, of course, as to how long to wait for transients to decay. 10000 data points appeared to be long enough for this regime for all but these few values of $\gamma$.
}

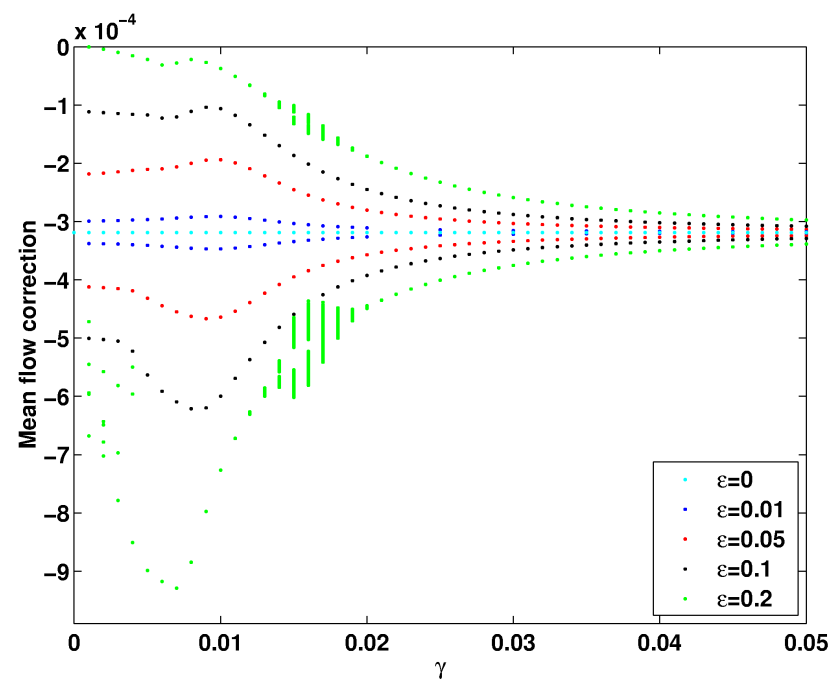

Fig. 5. The amplitude of the mean flow correction as a function of the forcing frequency $\gamma$ in the travelling wave regime. Experiments are conducted for several values of $\epsilon$.

subsequent such diagrams $X_{\min }$ is plotted in red and $X_{\max }$ in blue.

\subsubsection{Ramping $\gamma$ for fixed $\epsilon$}

Figure 6 shows $X_{\min }$ and $X_{\max }$ plotted as a function of $\gamma$ for $\epsilon=0.002$, i.e. a perturbation of $0.4 \%$ of the background flow. For most of the values of $\gamma$ the amplitude of the mean flow correction $X_{d}$ varies over a small range. There are two places, however, where it becomes constant, or varies between only two values, demonstrating period 1 and period 2 flow. ${ }^{4}$ They are marked on Fig. 6 . The period 1 flow arises around $\gamma_{n}$ as would be expected from synchronization theory (see e.g. Pikovsky et al., 2003); the period 2 flow is around $2 \gamma_{n} / 3$. As expected for the period 1 part of the flow $\frac{\omega}{\gamma}=\frac{1}{1}$ and for the period 2 part $\frac{\omega}{\gamma}=\frac{2}{3}$ - two of the so-called "Farey fractions". Examples of the flow for three different values of $\gamma$ are also seen in Fig. 6.

We now increase $\epsilon$ to 0.005 and again consider the flow as a function of $\gamma$, Fig. 7. The background flow is still only perturbed by $1 \%$. A notable feature here, compared to the $\epsilon=0.002$ case, is the period 2 flow which appears in the centre of the diagram. Synchronization theory (e.g. Pikovsky et al., 2003) predicts period 1 flow for small linear perturbations at frequencies around $\gamma_{n}$. We surmise here that although the forcing causes only a $1 \%$ variation in $U_{d}$, it is in a regime we shall label "moderate forcing" - it is not possible to completely predict the behaviour of the system analytically. Note also that a period 3 flow has also become visible, along with

\footnotetext{
${ }^{4}$ We mention only these two as they are most visible on the plot. There will be probably be others of course, depending on the resolution in $\gamma$ that we use.
} 


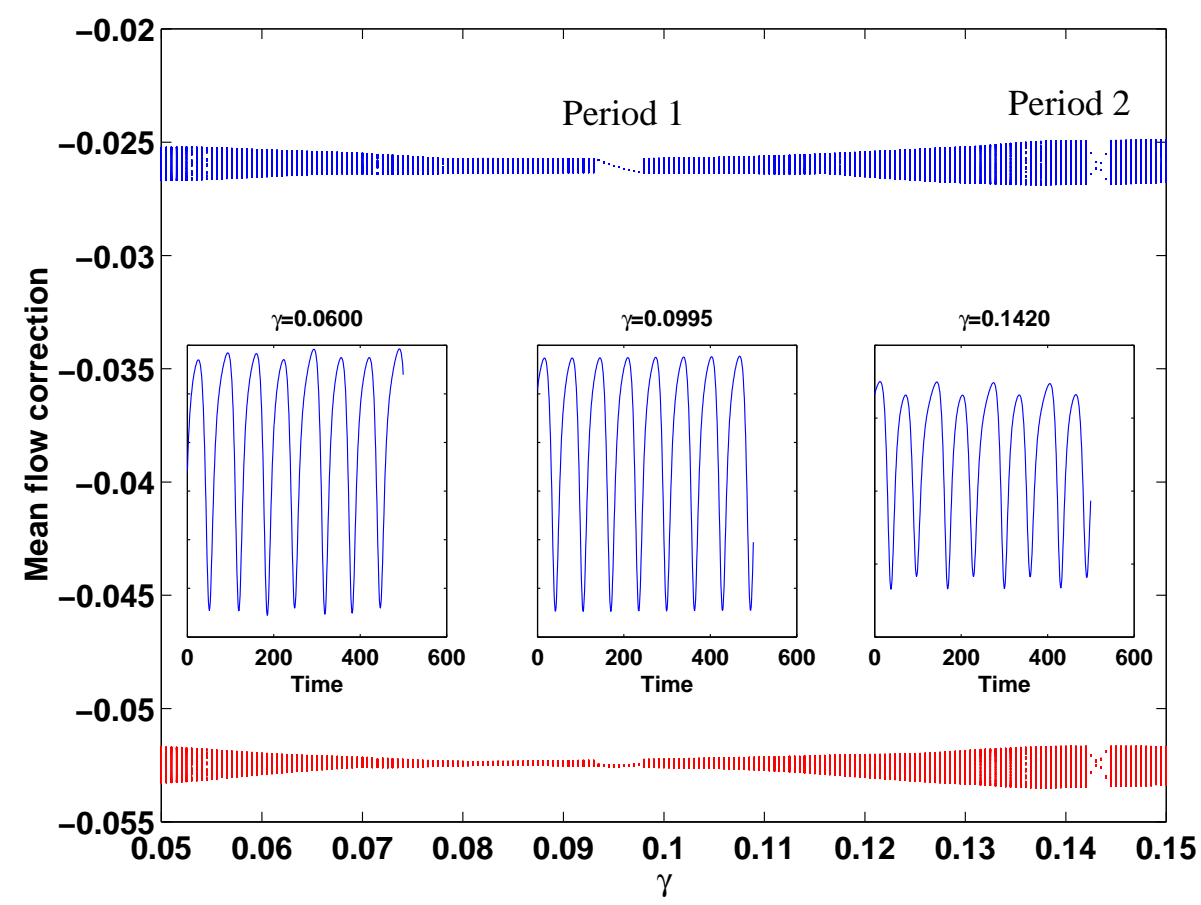

Fig. 6. $X_{\min }$ and $X_{\max }$ as a function of $\gamma$ for $\epsilon=0.002$ for the amplitude vacillation regime. Note $\gamma_{n}=0.0995$. The inserts show examples of the behaviour of $X_{d}$ as a function of time for 3 different values of $\gamma$. At $\gamma=0.06$ there is aperiodic behaviour, $\gamma=0.0995$ period 1 behaviour and $\gamma=0.142$ period 2 behaviour.

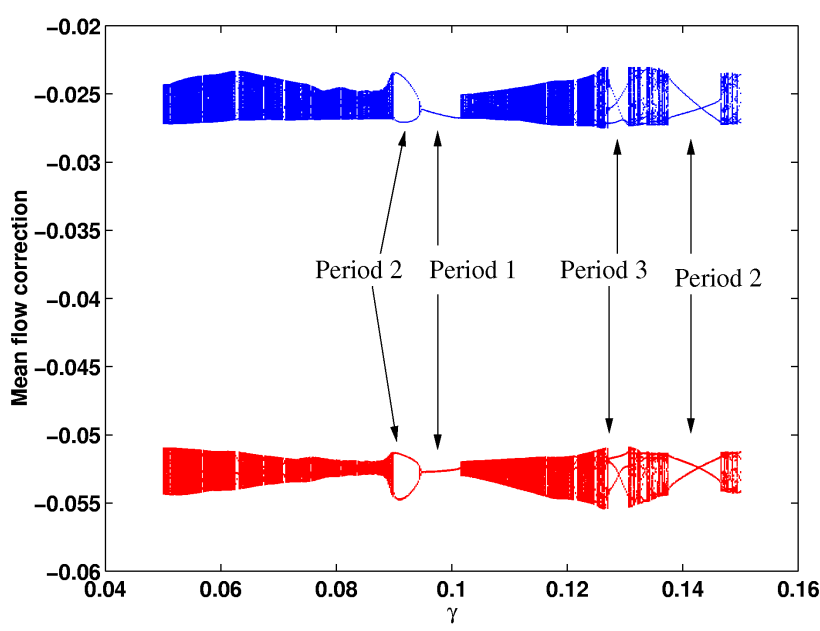

Fig. 7. $X_{\min }$ and $X_{\max }$ as a function of $\gamma$ for $\epsilon=0.005$ for the amplitude vacillation regime.

other smaller periodic windows. In these windows $\omega / \gamma$ can be written as $n / m$, where $n$ and $m$ are integers; it is evident we have a "devil's staircase" of frequency locking.

Examining this situation more closely we can observe the behaviour of the phase (Figs. 8 and 9) for a selection of $\gamma$ values. The phase difference, $\Delta \phi(t)$, slips just outside the central locked region (for $\gamma=0.0898$ ), though the histograms
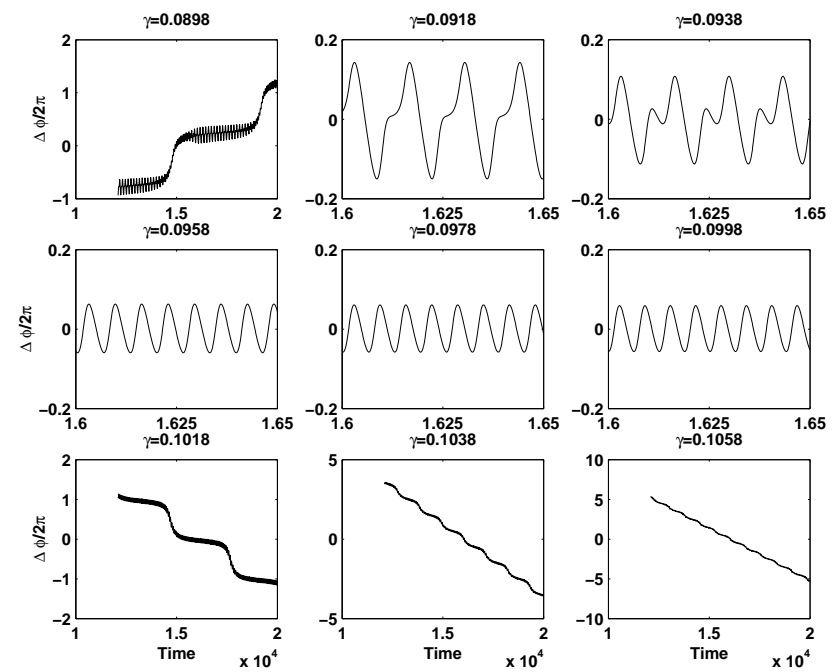

Fig. 8. Amplitude vacillation regime, $\epsilon=0.005$. Values of $\Delta \phi(t)$ for a range of $\gamma$. Note the changing ordinate. For $\gamma=0.0898$ there is quasi-periodic behaviour. Periodic behaviour of period 2 is seen for $\gamma=0.0918$ and 0.0938 . The middle three panels $(\gamma=0.0958,0.0978$, $0.0998)$ show period 1 . In the bottom three panels $(\gamma=0.1018$, $0.1038,0.1058)$ phase slips start appearing in $\Delta \phi(t)$, as we move to the edge of the Arnol'd tongue and back to a quasi-periodic state.

show broad peaks at certain values of $\Delta \phi$; for these values of $\gamma$ there is still a degree of partial synchronization. The 

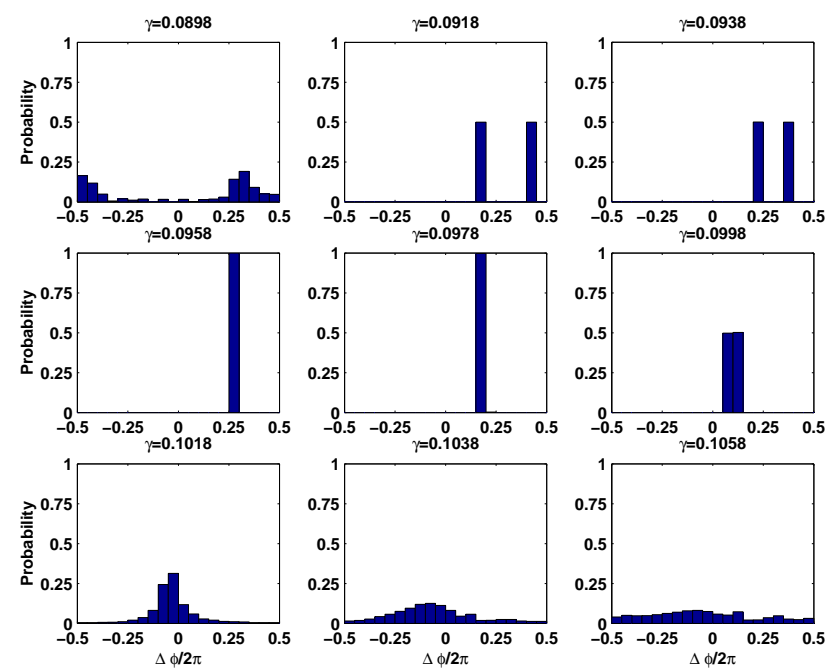

Fig. 9. Histogram of the data from Fig. $8, \Delta \phi(\bmod 2 \pi)$.

period doubling seen in the amplitude data, Fig. 7, can be seen also in the phase data $(\gamma=0.0918,0.0938)$, particularly in the histograms, as a point where $\Delta \phi$ clusters round two values. On the far side of the locked region $(\gamma=0.1018)$ the phase slips become increasingly frequent until $\Delta \phi(t)$ approaches a uniformly decreasing function, appropriate again to a quasi-periodic region $(\gamma=0.1058)$. The LLE for this run (not shown) indicates that the system remains largely non-chaotic until around $\gamma=0.13$ where it rises above zero to about $3 \times 10^{-3}$ bits per $\sigma$.

Similar runs for larger (constant) values of $\epsilon$ show a greater variation in $X_{\min }$ and $X_{\max }$, i.e. the underlying attractor is larger as the system explores more of the surrounding phase space (not shown). There are values of $\gamma$ for runs with $\epsilon=0.01$ and 0.05 , for which the LLE are significantly nonzero, thus indicating chaotic behaviour. Indeed the chaotic values of the LLE (i.e. the non-zero ones) are similar to those for the unperturbed chaotic system examined in the next section. Hence we label the forcing here as "strong".

Figure 10, for $\gamma$ ramped for a range of $\epsilon$, demonstrates the regions where there is periodic amplitude behaviour showing period $1,2 \ldots$ up to period 8 . For $\epsilon \leq 0.005$, which have largely non-chaotic behaviour for the whole range of $\gamma=0.05-0.15$, classic Arnol'd tongue-like behaviour can be seen; we can class the forcing here as "weak". The three main locked regions can be seen as the red tongue in the centre for $\gamma \approx 0.009$, the cyan tongue for $\gamma \approx 0.13$, and the green tongue $\gamma \approx 0.14$. At higher values of $\epsilon$ the middle tongue develops the period doubling discussed above, hence the tongue is not all in the same colour. Note the green and cyan tongues also develop period doubling in their centres. The three main synchronized regions also show up in the phase data (not shown), this time as places where $\frac{\omega}{\gamma}=\frac{n}{m}$.

For $\epsilon$ greater than $\sim 0.01$ the Arnol'd tongue is still visible in the amplitude data, Fig. 10, and to some extent in the

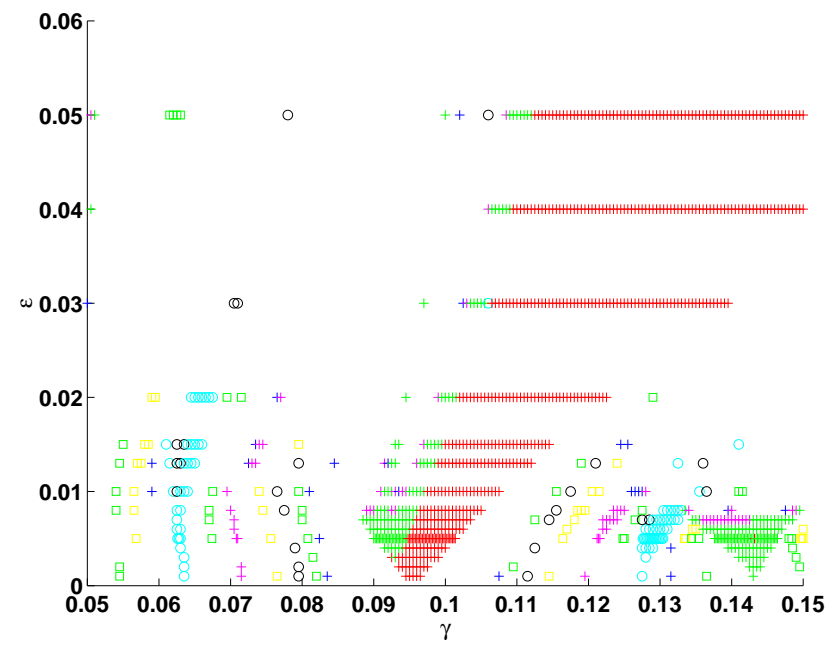

Fig. 10. The amplitude vacillation regime: plot of locked regions. Period $1+$, period $2+$, period 3 o, period $4+$, period 5 , period 6 o, period $7 \square$, period $8+$. Note the fields have not been contoured as they are highly complex and contouring may give a misleading impression.

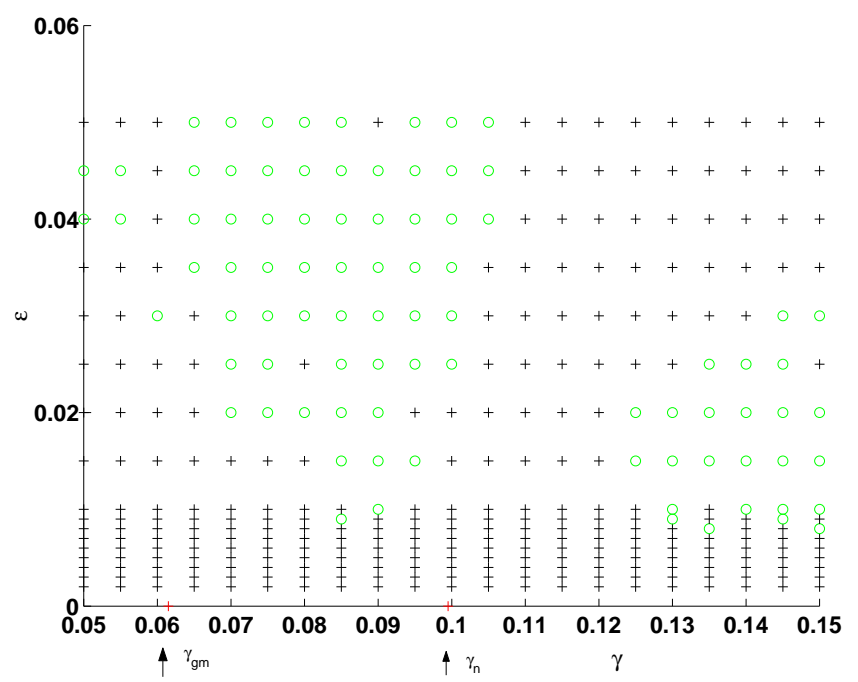

Fig. 11. The LLE as a function of $\gamma$ and $\epsilon$ for the amplitude vacillation regime. + denotes $L L E \leq 0.006$ and o LLE $>0.006$. The positions of $\gamma_{n}$ and $\gamma_{g m}$ (see text for definition) are marked. Again, as in Fig. 10, it was felt contouring the fields would be misleading.

phase data (not shown), though it is more skewed. Indeed for large values of $\gamma$ the 1:1 locked states do not even contain $\gamma_{n}$. Some of the previously locked states have disappeared altogether to be replaced by chaos. The system could be considered now to be jumping between the steps on the devil's staircase as they overlap, rather than remaining on any particular one, and so chaos instead of a coherent locked state is seen. The forcing amplitude is now classed as "large", and probably alters the underlying dynamics as well. 


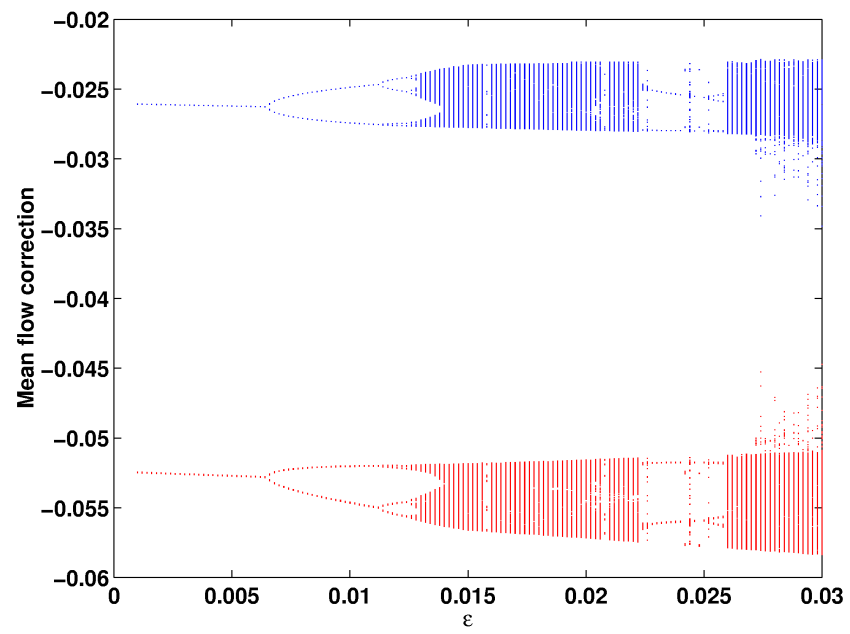

Fig. 12. $X_{\min }$ and $X_{\max }$ as a function of $\epsilon$, for $\gamma=\gamma_{n}$, in the amplitude vacillation regime.

Having considered the $(\gamma, \epsilon)$ parameter space from the point of view of the amplitude and of the phase, we present one more analysis, that of the LLE. The regions showing periodic behaviour of $X_{d}$ are, of course, all non-chaotic. However, it is possible to have regions where there is phase synchronization, but the dynamics (amplitude) remain chaotic (see Park et al., 1999; Zaks et al., 1999, for example). Figure 11 shows the regions where the $\operatorname{LLE}<0.002$, i.e. $\sim 0$ (shown in black) and regions where the LLE $>0.002$ (shown in blue). For values of $\epsilon<0.005$, the system is non-chaotic for all the frequencies of forcing that we investigated. For the lowest value of $\epsilon$ for which chaos was observed, i.e. for $\epsilon=0.005$, the $\gamma$ required is not close to $\gamma_{n}$ as was found by Franz and Zhang (1995), for example, for the real Lorenz equations. Instead it is at $\gamma \sim 0.13$. Chaos also set in for low values of $\epsilon$ around $\gamma \sim 0.085$. The fact that the tongue of stability is skewed leads to the following interesting result. For low values of $\gamma$, i.e. $\gamma<\gamma_{n}$, the range in $\gamma$ for which chaos is observed, increases with increasing forcing amplitudes. For high values of $\gamma$ the converse is true. For very low amplitudes of forcing the system is, as we said above, non-chaotic. However it quickly becomes chaotic at moderate forcing amplitudes and then becomes non-chaotic again at high forcing amplitudes. If we denote the detuning $v$, as $\nu=\gamma-\gamma_{n}$, it would seem that the chaotic behaviour for moderate and large forcing amplitudes depends on the sign of the detuning, as well as its magnitude. There is also a small range of $\gamma$ for which the system remains stable for all the forcing amplitudes we investigated.

\subsubsection{Ramping $\epsilon$ for fixed $\gamma$}

The investigations of the previous section allowed an examination of the behaviour of the periodic regime over a range of forcing parameters $\gamma$ and $\epsilon$. Before leaving this regime

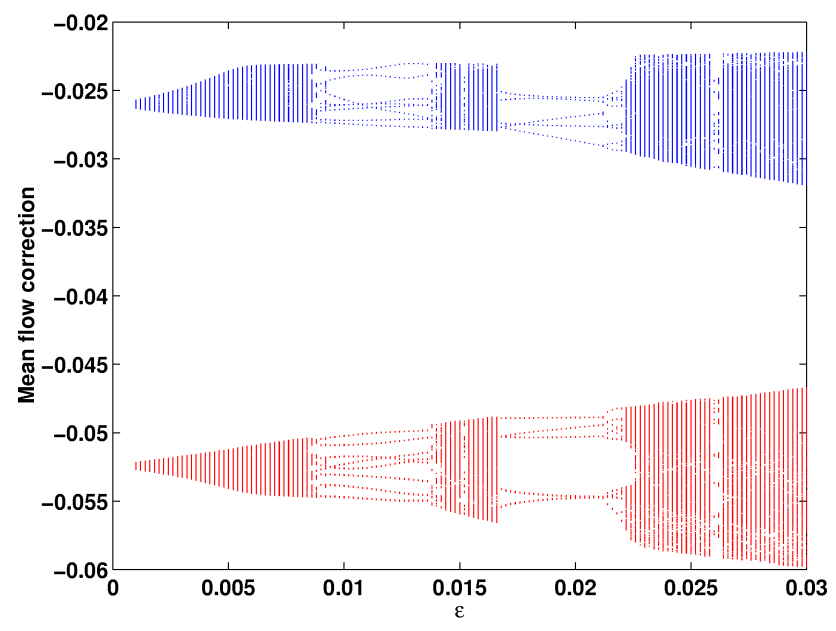

Fig. 13. $X_{\min }$ and $X_{\max }$ as a function of $\epsilon$ in the amplitude vacillation regime for $\gamma=\gamma_{g m}$ (for definition see text).

we study further two particular points in parameter space, this time holding $\gamma$ fixed. We consider $\gamma=\gamma_{n}$, and $\gamma$ as far away as possible from any frequency rationally related to $\gamma_{n}$. Hence we consider the natural frequency, $\gamma_{n}$, multiplied by the golden mean. ${ }^{5}$ Thus we define $\gamma_{g m}=\frac{\sqrt{5}-1}{2} \gamma_{n}$.

Consider first the case for $\gamma=\gamma_{n}$, Fig. 12. For small values of $\epsilon$ the system continues to oscillate at its natural frequency, showing period 1 behaviour. However, there follows a period doubling route to a chaotic state, with various different periodic states visible as $\epsilon$ increases further. Thus we can see where the classic Arnol'd tongue-like behaviour breaks down, though further locked states become apparent at higher values of $\epsilon$ (e.g. around 0.022). The onset of chaos can also be seen in the behaviour of the LLE in Fig. 11. Studying the phase behaviour (not shown) shows $\Delta \phi(t)$ remains bounded for all time, even though the distribution of $\Delta \phi$ may vary and the specifics of its time dependence varies.

Figure 13 shows the case for $\gamma=\gamma_{g m}$. Now $\epsilon$ must be greater than $\sim 0.008$ before any locking emerges. Interestingly the LLE remains around zero for the whole of this run, indicating that this state is largely quasi-periodic rather than chaotic over the range of $\epsilon$ shown. The location of $\gamma_{g m}$ is marked on Fig. 11. Thus forcing at a frequency far away from a frequency rationally related to the natural frequency presumably requires a large driving amplitude (larger than in any of our runs) to cause a transition to chaotic behaviour.

\subsection{The chaotic regime}

In Sect. 2.2.5 we found that there were UPOs present in the chaotic regime at periods of $\tau_{f} \approx 40.45 \sigma\left(\gamma_{f}=0.1553\right)$, and

\footnotetext{
${ }^{5}$ The golden mean, $p$, can be found as a root of the equation, $1+\frac{1}{p}=p$. It is a continued fraction, i.e. $p=1+\frac{1}{p}=1+\frac{1}{1+\frac{1}{p}}=\ldots$ etc.
} 


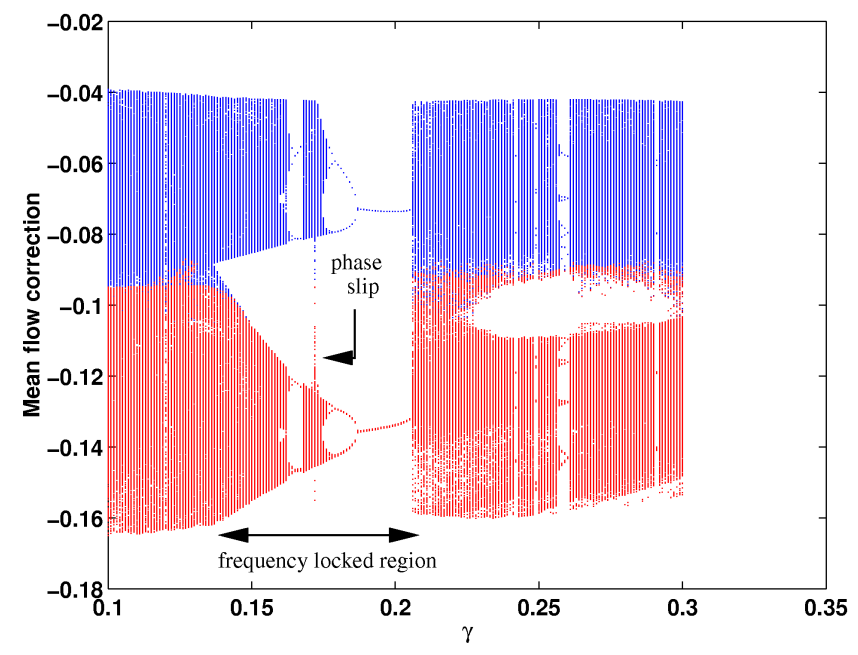

Fig. 14. Application of forcing with $\epsilon=0.05$ in the chaotic regime. The position of the phase slip corresponds to $\gamma=0.172$ in Fig. 15 . See text for further details.

integer multiples of this, and hence we shall pay particular attention to forcing at these periods. For all runs, the parameter space of the unforced system that was visited corresponded to a chaotic region, and hence any ensuing periodicity is due to the addition of forcing rather than crossing a bifurcation point of the unforced system. (It could also be due to the bifurcation point moving as a result of the forcing, but even then the unforced system has been changed.)

\subsubsection{Ramping $\gamma$ for fixed $\epsilon$}

First the value of $\epsilon$ is held fixed for each experiment, and the value of $\gamma$ ramped from 0.1 to 0.3 . Runs were also carried out ramping $\gamma$ from 0.005 to 0.1 , though in most runs the forcing had little effect on the chaotic behaviour so they are not shown.

Forcing with $\epsilon=0.01$ has little effect and the LLE, remains fairly constant (and positive) throughout. When the amplitude of oscillation is increased to $\epsilon=0.02$, small windows appear where the chaotic behaviour disappears to be replaced by regular amplitude vacillation, and the corresponding LLE drops to zero. As $\epsilon$ is increased still further the windows of regular periodic behaviour increase in their range in $\gamma$.

For example the case of $\epsilon=0.05$ is shown in Fig. 14, with a region of period 2, going through an inverse period-doubling cascade to period 1. Examining the ratios of $\omega / \gamma$ for this run shows that the region of frequency locking (i.e. synchronization) extends beyond the bounds of the region of amplitude periodicity (see Figs. 16 and 17 later). This locked region is marked on Fig. 14; note that it corresponds to the region of decreased variation in amplitude.

An example of the behaviour of $\Delta \phi(t)$ going into and out of the locked region for $\epsilon=0.05$ can be seen in Fig. 15 . Phase slips are seen in Fig. 15, going into the locked re-
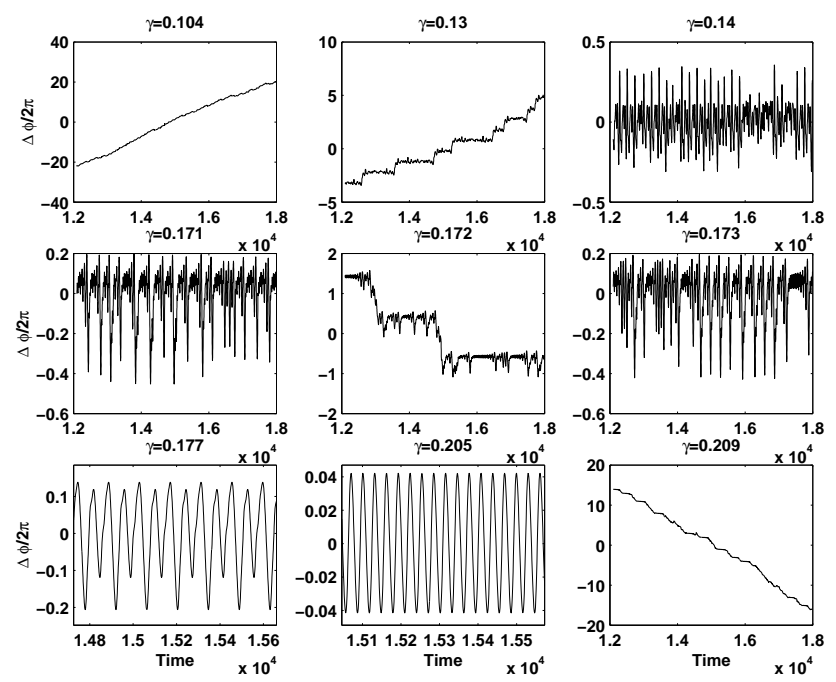

Fig. 15. $\Delta \phi(t)$ for the chaotic regime with $\epsilon=0.05$. Panels $3-8$ correspond to the frequency locked region maked on Fig. 14. Note $\Delta \phi(t)$ remains bounded though its precise behaviour varies. The phase slip seen in panel 5 is also marked on Fig. 14.

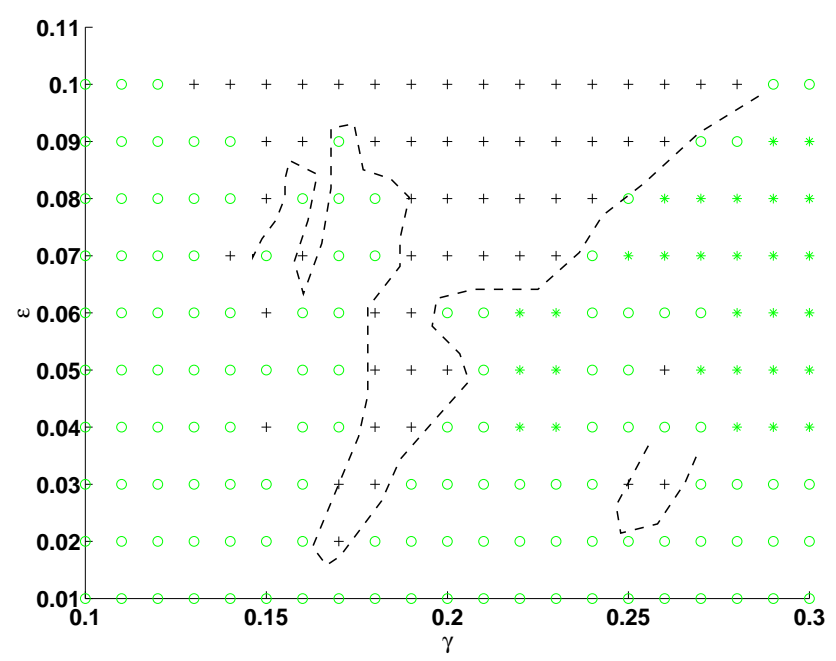

Fig. 16. The variation of LLE with $\gamma$ for various values of $\epsilon$ in the chaotic regime. + delineates $\mathrm{LLE}<0.002$, o $0.002 \leq \mathrm{LLE}<0.03$ and $*$ LLE $\geq 0.03$. The non-chaotic behaviour is therefore given by the black regions, and chaotic behaviour is seen in the green regions.

gion, in the same way as in the periodic regime (e.g. $\gamma=0.13$ ). However, on examining the phase behaviour in Fig. 15 over this frequency locked region $(\gamma=0.14-0.205)$ it can be seen that it varies quite considerably. There are regions where $\Delta \phi(t)$ behaves in an erratic but bounded way (e.g. $\gamma=0.14$, $0.171,0.173)$, regions where it oscillates in a regular way (e.g. $\gamma=0.177,0.205$ ), and even one where it performs phase slips $(\gamma=0.172)$. The corresponding phase-slip is marked on Fig. 14. The PDFs (not shown) all have distinct peaks in this region showing synchronization is taking place. Hence there 


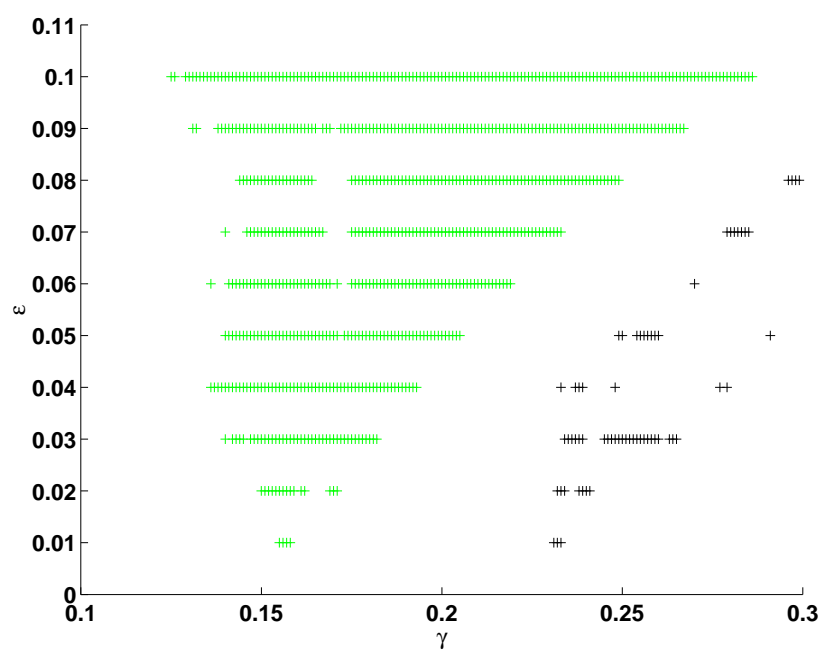

Fig. 17. The chaotic regime: the values of $\epsilon$ and $\gamma$ for which the ratio $\omega / \gamma \approx 1$ are shown in green and $\omega / \gamma \approx 2 / 3$ are shown in black.

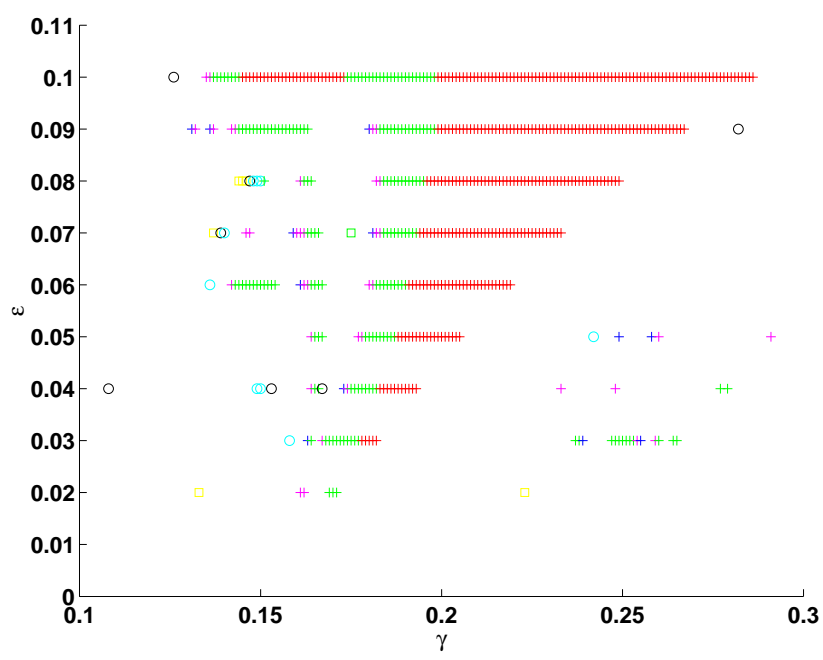

Fig. 18. The chaotic regime: plot of amplitude locked behaviour. Period $1+$, period $2+$, period 3 o, period $4+$, period 5 , period 6 o, period $7 \square$, period $8+$.

are regions where frequency entrainment is seen, where the amplitude behaviour is still chaotic, and $\Delta \phi(t)$ is also irregular though bounded.

The LLE (not shown) demonstrates increasing the amplitude of the forcing from 0.01 to 0.1 seems to have two main effects. The width of the windows of locked, regular periodic behaviour increases, but also, in the chaotic regions, the LLE itself in the chaotic states increases with increasing $\epsilon$, particularly for the higher values of $\gamma$. A summary of the LLE behaviour over a range of parameters is seen in Fig. 16. The periods of regular behaviour (shown in black) and increased chaotic behaviour (shown in red) can be clearly seen. The cut-off point between the blue and red areas of the plot is somewhat arbitrary; a value of LLE $=0.03$ was chosen; the unforced system has an LLE of $\sim 0.02$.

We now compare Fig. 16 with Fig. 17. The first shows the region where the LLE drops below a certain threshold value, i.e. is $\approx 0$, and the system is behaving in a non-chaotic way. The second of these two shows regions where $\omega / \gamma=1$ and $2 / 3$ (recall $\omega=\dot{\phi}(t)$ ); this shows regions of frequency locking. The two are not identical. This is because, as was pointed out earlier, it is possible to have frequency locking in a chaotic system, without necessarily having the amplitude also behaving in a regular way. As Pikovsky et al. (1997) point out, the LLE alone cannot be used to determine the point of phase synchronization, as this can occur in regions where the overall behaviour of the system is still chaotic.

Further, it is also interesting to note the frequencies for which stability occurs. From the results of Mirius and Sprott (1999) we would expect this to be at the periods of the UPOs or integer multiples of these. The period one UPO has $\gamma_{f}=0.15533$, and the main region of stability for all the values of $\epsilon$ considered here has period one (as seen in the amplitude bifurcation plots), born from a period-two inverse period-doubling cascade. However, these are clearly stablised not just at $\gamma=\gamma_{f}$, but over a range in $\gamma$, the range depending on the value of $\epsilon$. Examining Fig. 16, the required $\gamma$ for periodic behaviour, for the smallest value of $\epsilon$, seems to be $\sim 0.17$, with the range of $\gamma$ values increasing with increasing $\epsilon$, though for some values of $\epsilon$ this value of $\gamma$ does not give a non-chaotic system. Hence this seems not to be in agreement with the findings of Mirius and Sprott (1999) for the real Lorenz system. The reasons for this are not entirely clear and merit further investigation.

The plot of the frequency locked regime (for 1:1 locking), however, does seem to tend to $\gamma=\gamma_{f}$ as $\epsilon \rightarrow 0$ (see Fig. 17), though the tongue subsequently is not symmetric about this value for increasing $\epsilon$. Thus, forcing at the fundamental UPO period, at very low amplitudes, does seem to control the phase behaviour of the system (and more so its average frequency), although the same is not true for the amplitude as we stated above.

We have considered the overall behaviour of the system from the perspective of Lyapunov exponents and the chaotic/non-chaotic behaviour of the system (Fig. 16), and we have considered it from a frequency locking perspective (Fig. 17). A third possible view, as we used in the amplitude vacillation regime, is to examine the periodicity of the amplitude in the locked regions. This is shown in Fig. 18. Again the main tongue of regular behaviour is seen almost solely at period 1 and period 2 (in contrast to the AV plot in Fig. 10). A second area of periodic behaviour, predominantly at period 2 at about $\gamma=0.25$, is also seen where the $2 / 3$ frequency locking regime occurs, similar to the periodic case.

Kociuba and Heckenberg (2002), similarly, find various locking ratios and amplitude periodicities for their version of the complex Lorenz equations for a laser with periodic forcing. Their main $1 / 1$ locked tongue has period 3 behaviour 


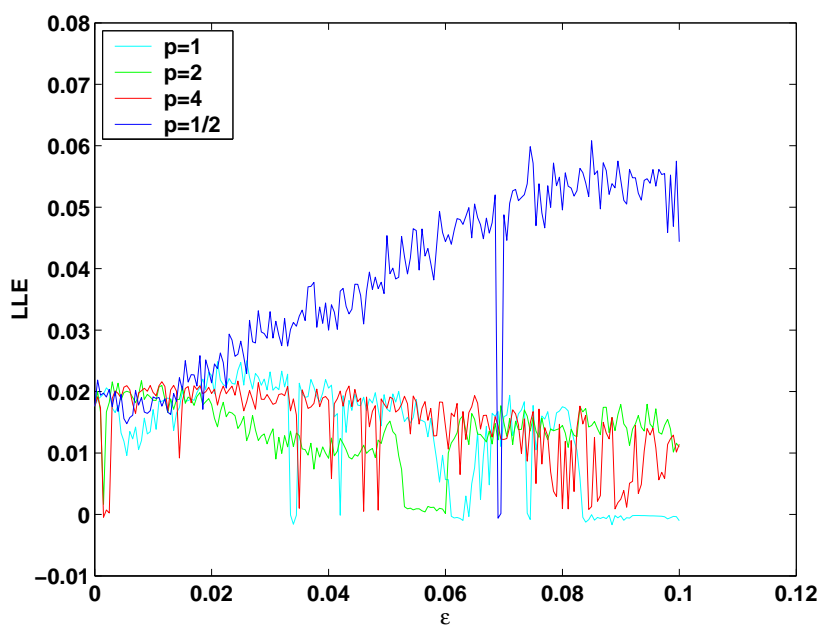

Fig. 19. The variation in LLE with $\epsilon$ for various frequencies of forcing in the chaotic regime.
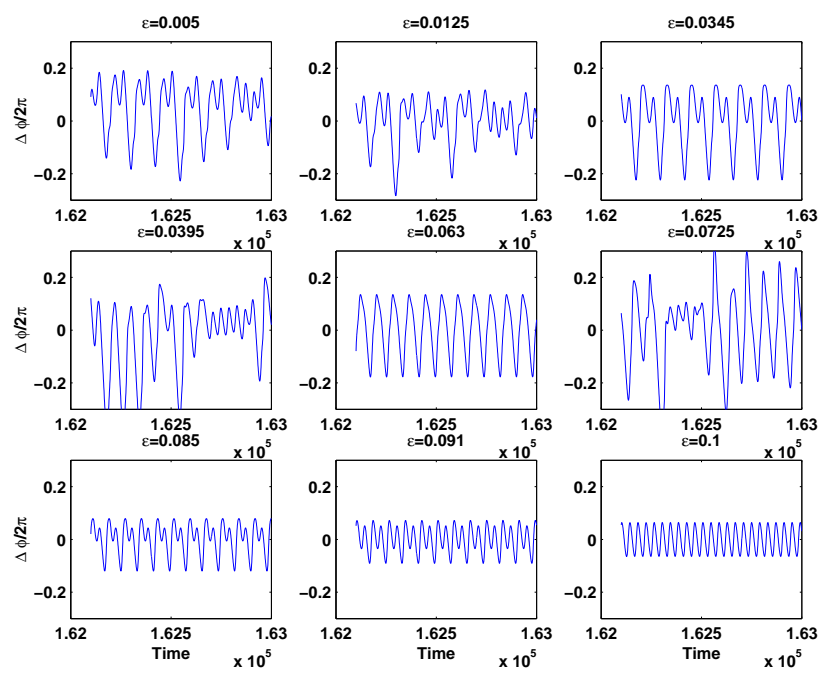

Fig. 20. $\Delta \phi(t)$ for a range of $\epsilon$ forcing at $\gamma=\gamma_{f}$ in the chaotic regime. The phase difference remains bounded throughout this run.

(of the amplitude) in the centre, with period 2 and period 4 visible on either side. This is in contrast to ours which is predominantly period 1 , with period 2 on one side. The behaviour is qualitatively similar, however.

\subsubsection{Ramping $\epsilon$ for fixed $\gamma$}

As with the AV regime studies, we now pick out particular values of $\gamma$ for further analysis, fixing the forcing at these values of $\gamma$ and ramping the amplitude. We consider forcing at periods which are integer multiples of the fundamental UPO period, and at other periods as explained below.
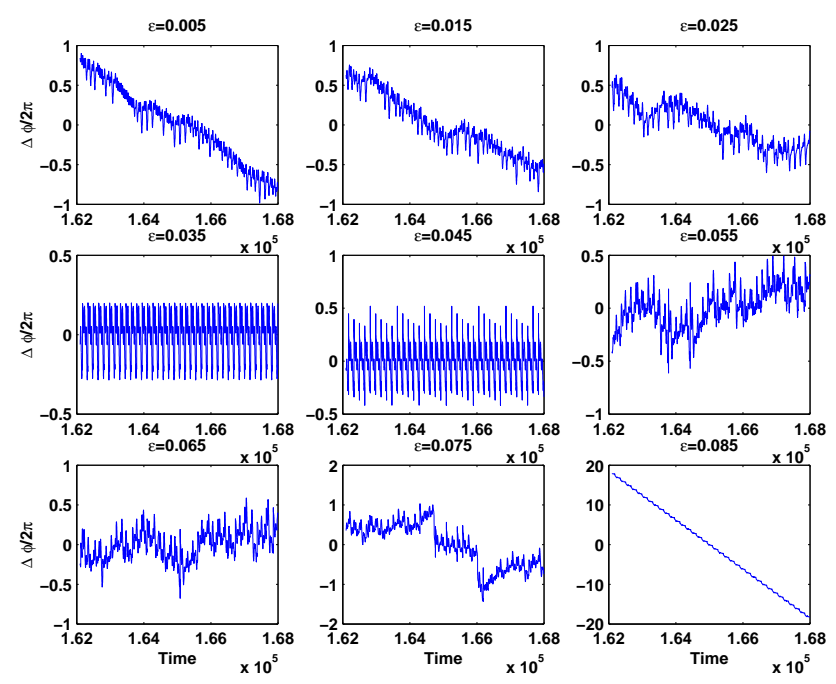

Fig. 21. $\Delta \phi(t)$ for a range of $\epsilon$ forcing at $\gamma=\gamma_{f} / 4$. Panels 4 and 5 are regions of complete phase locking where the corresponding histogram (not shown) is a single sharp peak.
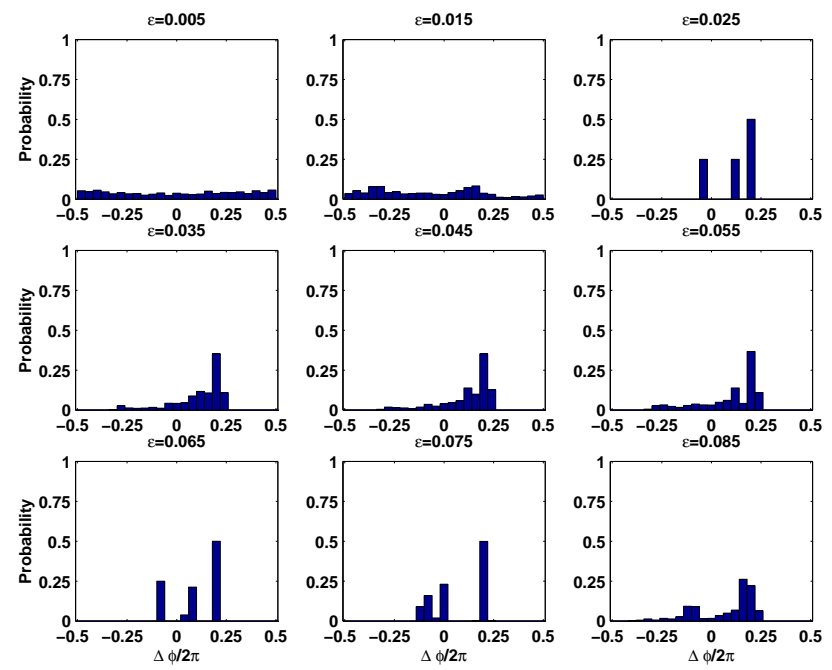

Fig. 22. The histogram for a range of $\epsilon$ forcing at $\gamma=0.161$, in the chaotic regime. There is some degree of synchronization for many values of $\epsilon$.

\section{Forcing at the UPO periods}

First we consider forcing at frequencies equal to one, two, three, four and half the fundamental UPO period, i.e. at $\gamma_{f} / p$, where $p=1,2,3,4,0.5$, respectively. In all these cases the system remains largely chaotic, though there are a few windows of regular periodic behaviour, as shown by the LLE in Fig. 19. The $p=1 / 2$ case is notable because there is very little stability and the LLE increases steadily, whereas for the $p=1-4$ cases the LLE remains less than $\sim 0.02$ bits $/ \sigma$. 
In the $p=2,3,4$ cases the largest windows of periodic behaviour are at period two, three and four, respectively, as we might expect (not shown). In contrast, forcing at the $p=1$ frequency, gives the main regions of periodicity being about half at period one and half at period two. The period doubling is present here too (not shown).

Examining the behaviour of $\Delta \phi$ when forcing at $\gamma_{f}$ (Fig. 20) shows that the phase remains bounded for the full range of forcing amplitudes, and the histograms (not shown) are all sharply peaked. Forcing at this frequency certainly seems to strongly affect the behaviour, showing (at least partial) synchronization, though the specific behaviour varies as $\epsilon$ varies. Only in certain cases do we have phase synchronization in the strictest sense, e.g. for $\epsilon=0.063$. Contrast this with the behaviour for the forcing at four times the fundamental UPO period. Anticipating interaction around $\omega / \gamma=4$, we calculate the phase difference this time as

$\Delta \phi(t)=\phi_{x}(t)-4 \gamma t$.

The results can be seen in Fig. 21. There are some values for which phase synchronization is seen (e.g. panels 4 and $5)$, but for others $\Delta \phi(t)$ is unbounded and increases or decreases with time.

\section{Forcing at non-UPO periods}

Finally a non-UPO frequency of oscillation was chosen by studying the results from the ramping $\gamma$ runs, choosing a value of $\gamma$ that gave periodic behaviour, $\gamma=0.161$. The phase behaviour at this value suggests that this frequency is effective at controlling the system to some extent since $\Delta \phi(t)$ is bounded for a large range of $\epsilon$. There is a strong phase preference at certain $\epsilon$ values (see Fig. 22) indicating a degree of synchronization. Examination of the frequency data suggests that $\gamma=0.161$ has regions with locked ratios of $\omega / \gamma=1 / 4$. Hence the "natural period" here would correspond to $\tau=2 \pi / 0.161 \times 4=156$. It is notable that the histogram of UPO periods in Fig. 4 has a very small peak at this period. Hence controlled behaviour may be expected by forcing periods which are rationally related to this. Indeed similar controlled regions could be found by studying the other small peaks of the UPO histogram.

\section{Sensitivity to initial conditions}

Interestingly, when comparing the LLEs for these two sets of experiments, ramping $\gamma$ and ramping $\epsilon$, for the values of $(\gamma, \epsilon)$ common to both, the two do not always agree. In other words there are regions of parameter space in which the results are more sensitive to initial conditions. Mirius and Sprott (1999) comment that differing conditions led to differing specific trajectories in their studies on the Lorenz equations, but not different behaviour. Kociuba and Heckenberg (2002), however, found that for some values of perturbation frequency and amplitude control did not occur for all initial conditions in their studies on the Lorenz equations with complex coefficients.

It is certainly true for our system, as for that of Mirius and Sprott (1999), that as the amplitude of the perturbation is increased at a fixed frequency, the system can pass through regions of control and chaos. This is unlike the classic Arnol'd tongue type behaviour seen in Sect. 3.2 in the amplitude vacillation regime for small $\epsilon$. Here, once locked, the system remains so as $\epsilon$ is increased. However the changing from controlled to chaotic regions is more like the large $\epsilon$ behaviour seen for the amplitude vacillation regime, where the tongue behaviour was less regular and in fact chaotic behaviour was possible even when forcing at $\gamma_{n}$.

\section{Discussion and conclusions}

Applying a periodic forcing to a quasi-periodic or chaotic system can significantly alter the behaviour of the system. In the climate community this effect is of great interest with respect to oscillatory phenomena such as ENSO, the QuasiBiennial Oscillation or Tropical Instability Waves, for example, and their interaction with annual or sub-annual solar forcings. Here we have considered one of the simplest geophysical fluid systems: a two-layer quasi-geostrophic model, allowing one wave in the along-stream and cross-stream directions. The bifurcation structure and unforced behaviour of this model has been extensively mapped by Lovegrove et al. (2001, 2002). Here we applied periodic forcing by directly varying the baroclinic velocity field in a sinusoidal manner. Although the choice of parameters for this study has necessarily been limited, we have, nonetheless, found a rich set of solutions.

We have concentrated on three different regimes identified by Lovegrove et al. (2001, 2002): a steady, travelling wave regime, a quasi-periodic, modulated wave regime and a chaotic regime. First we showed that when a periodic forcing is applied to a steady travelling wave regime, a resonance is seen, albeit of small amplitude, when there is no such stable "natural frequency" of the system in the absence of forcing; see Fig. 5. Note Pedlosky and Thomson (2003) also found an oscillatory state in a previously sub-critical regime when a periodic forcing was applied.

A summary of the regime diagrams for the periodic and chaotic flows, plotted schematically as functions of $(\gamma, \epsilon)$, was presented in Fig. 1. The fundamental UPO frequency, $\gamma_{f}$, in the chaotic regime appears to play a similar role to that of the natural frequency, $\gamma_{n}$, in the periodic regime. We summarise some of the behaviour seen in these two regimes below.

In the periodic regime, at small amplitudes of forcing, the classic Arnol'd tongues were seen, with Farey ratios of frequency locking, periodic behaviour in the amplitude, and a devil's staircase. At moderate forcing amplitudes, period doubling cascades were seen in the amplitude behaviour (and 
indeed in the phase behaviour), at the edge of the three main Arnol'd tongues. Phase slips were also seen at the edge of the periodic windows. Most of the periodic amplitude behaviour of higher periodicities disappeared at these moderate forcing values, as did some of the higher order frequency locked regimes. The amount of chaotic behaviour (i.e. the range of $\gamma$ for which the LLE $>\sim 0.003$ ) increased with increasing $\epsilon$ (Fig. 11), and the Arnol'd tongue became highly asymmetric with respect to the detuning on either side of the tongue. Pedlosky and Thomson (2003) also found that the stronger the periodic forcing the more likely chaotic behaviour was to ensue when the shear was varied periodically in slightly supercritical (when unforced) conditions. Our results were not similar however to those of Franz and Zhang (1995), for example, who found that forcing at the natural frequency was most likely to induce chaos in the real Lorenz equations. However, we did agree with them in the sense that a frequency not rationally related to the natural frequency, $\gamma_{g m}$, showed no chaotic behaviour for the whole range of forcing amplitudes used in this study. Throughout the investigations period 1 behaviour was frequently born out of an inverse period doubling cascade.

In the chaotic regime the chaos was suppressed for particular values of $\gamma$ and the region over which this suppression took place increased with increasing $\epsilon$, as seen by the black tongue of Fig. 16. This tongue however has "holes" in it, in the form of regions where the chaos returns. Moreover not all points that are stable have stability for all initial conditions.

Instead of searching for total synchronization, we can instead observe frequency locking, with the frequency of the system being defined as the time mean value of $d \phi_{x} / d t$. Then the regions of synchronization are more extensive, see Fig. 17.

The system can also be studied from the perspective of amplitude periodicity with a variety of periodic behaviours of the amplitude visible in the main central frequency-locked, non-chaotic tongue, in qualitative agreement with the findings of Kociuba and Heckenberg (2002) studying the complex Lorenz equations for a laser, Fig. 18.

Our results are generally consistent with those of Mirius and Sprott (1999) in that forcing at a period equal to the principal UPO period was likely to give rise to total synchronization, though not for all values of $\epsilon$. A forcing period of $4 \tau_{f}$ was also particularly successful at quenching chaos, as might be expected from the histogram of UPOs, Fig. 4. From a phase synchronization perspective we found that forcing at the principal UPO period led to restricted ranges of $\Delta \phi(t)$ for almost all values of $\epsilon$ and to complete phase locking in some cases. Forcing at the other UPO periods did cause phase locking and synchronization, but to a lesser extent, defining the value of $\Delta \phi(t)$ appropriate to the particular multiple of the fundamental UPO in each case. For total synchronization at the smallest possible value of $\epsilon$, control seemed to be achieved not for the fundamental UPO period, but for a non harmonically related value of $\gamma \sim 0.17$. Frequency locking is possible for smaller $\epsilon$ values than this, however, and the region of locking $i s$ then consistent with the principal UPO period of $\tau_{f}=40.45$. Again, similar to the AV regime, period one locking is usually born out of an inverse period doubling sequence.

Returning finally to the climate, there remains the question of the applicability of these results on a highly idealised system to that of a real geophysical system. Clearly, their applicability to climate cycles, for example, is tenuous. Indeed, the highly truncated model of baroclinic instability due to Klein and Pedlosky (1986) shows strong dependence on spectral resolution. We therefore doubt that our solutions have quantitative significance for climate but rather illustrate the range of possible behaviour. The most obvious distinction between the present model and the real geophysical system is of complexity and state-space dimension. The real system is vastly more complex than our 5-dimensional model. It is therefore natural to suppose that the real system exhibits richer and more varied behaviour than we see here. This is an intriguing prospect because the present system already displays great subtlety in response to periodic forcing. Indeed, our results are necessarily somewhat incomplete owing to this complexity. The forcing function used here is monochromatic and noise free. Perhaps extrapolation of our results to geophysical systems should therefore be restricted to similar cases. In practice, this would mean external periodic forcing whose origin is in the Earth's celestial mechanics (e.g., tides, seasonal cycle, Milankovitch cycles). In any event, it is of interest to speculate a little about how syncronization phenomena may be at work in geophysical, and laboratory, rotating, stratified fluids.

At least some aspects of the index cycle, NAO and associated annular modes, have analogues in the rotating annulus experiments. This Q-G model is the simplest representation of such a laboratory system. Our results would suggest that certain aspects of the northern hemisphere variability could be due to interactions with a periodic forcing, be it annual or with another period, in line with one proposal by Stephenson et al. (2000). However the nature of the forcing in the atmosphere is not mono-periodic so furthering the analogy requires the addition of further forcing frequencies to the model and possibly stochastic terms as well.

Recent climate work has also focussed on much shorter timescales examining intraseasonal variability. Including a seasonal cycle in GCMs can improve representations of intraseasonal tropical oscillations such as the Madden-Julian oscillation (Huang and Cho, 1998). Moving to the extratropics, modes with periods of around 20-40 days have been found in both hemispheres, with wavenumbers 4-7 (e.g. Randel and Stanford, 1983, 1985b; Ghil and Mo, 1991a,b). Possible analogous phenomena occur in the laboratory in the multiple wave regimes, both with and without periodic forcing. This motivates further work on this Q-G model, but including more than one azimuthal wave. The results with only one wave, as presented here, suggest that if there was a 
forcing with a varying amplitude, the atmosphere could flip between chaotic and stable states perhaps giving rise to some of the observed features.

Thus the subtle, complex behaviour in the low-order system we study here might have analogues in some periodically-forced geophysical phenomena. Further work on the applicability of these results from low-order systems to climate variability is clearly required.

Acknowledgements. Many thanks to I. Moroz for her helpful suggestions. Thanks too to the two anonymous referees. F. J. R. Eccles was supported by a NERC studentship and grants from Lincoln College and The University of Oxford.

Edited by: R. Grimshaw

Reviewed by: Two referees

\section{References}

Allen, M., Lawrence, S., Murray, M., Mutlow, C., Stockdale, T., Llewellyn-Jones, D., and Anderson, D.: Control of tropical instability waves in the Pacific, Geophys. Res. Lett., 22, 2581-2584, 1995.

Baldwin, M., Gray, L., Dunkerton, T., Hamilton, K., Haynes, P., Randel, W., Holton, J., Alexander, M., Hirota, I., Horinouchi, T., Jones, D., Kinnersley, J., Marquardt, C., Sato, K., and Takahashi, M.: The quasi-biennial oscillation, Rev. Geophys., 39, 179-229, 2001.

Benestad, R., Sutton, R., Allen, M., and Anderson, D.: The influence of subseasonal wind variability on tropical instability waves in the Pacific., Geophys. Res. Lett., 28, 2041-2044, 2001.

Braimin, Y. and Goldhirsch, I.: Taming chaotic dynamics with weak periodic perturbations, Phys. Rev. Lett., 66, 2545-2548, 1991.

Ding, W. X., She, H., Huang, W., and Yu, C.: Controlling chaos in a discharge plasma, Phys. Rev. Lett., 72, 96-99, 1994.

Fowler, A., Gibbon, J., and McGuinness, M.: The complex Lorenz equations, Physica D, 4, 139-163, 1982.

Franz, M. and Zhang, M.: Supression and creation of chaos in a periodically forced Lorenz system, Phys. Rev. E, 52, 3558-3566, 1995.

Fronzoni, L., Giocondo, M., and Pettini, M.: Experimental evidence of suppression of chaos by resonant parametric perturbations, Phys. Rev. A, 43, 6483-6487, 1991.

Früh, W.-G. and Read, P.: Wave interactions and the transition to chaos of baroclinic waves in a thermally driven rotating annulus, Phil. Trans. R. Soc. London, 355, 101-153, 1997.

Gabor, D.: Theory of Communication, J. IEE (London), 93, 429457, 1946.

Geller, M., Shen, W., Zhang, M., and Tan, W.-W.: Calculations of the stratospheric quasi-biennial oscillation for time-varying wave forcing, J. Atmos. Sci., 54, 883-894, 1997.

Ghil, M. and Le Treut, H.: A climate model with cryodynamics and geodynamics, J. Geophys. Res., 86, 5262-5270, 1981.

Ghil, M. and Mo, K.: Intraseasonal oscillations in the global atmosphere. Part I: Nothern Hemisphere and Tropics, J. Atmos. Sci., 48, 752-779, 1991a.

Ghil, M. and Mo, K.: Intraseasonal oscillations in the global atmosphere. Part II: Southern Hemisphere, J. Atmos. Sci., 48, 780790, $1991 b$.
Hart, J.: A laboratory study of baroclinic instability, Geophys. Fluid. Dyn, 3, 181-209, 1972.

Hart, J.: Finite Amplitude baroclinic instability with periodic forcing, Physica D, 39, 239-261, 1989a.

Hart, J.: The modulated complex Lorenz equations: baroclinic instability with seasonal forcing on the $\beta$-plane, Summer Study Program in GFD, WHOI, 1989b.

Hide, R. and Mason, P.: Sloping convection in a rotating fluid, Adv. Phys., 24, 47-100, 1975.

Higuchi, K., Huang, J., and Shabbar, A.: A wavelet characterization of the North Atlantic Oscillation variation and its relationship to the North Atlantic sea surface temperature, Int. J. Climatol., 19, 1119-1129, 1999.

Huang, J.-P. and Cho, H.-R.: Seasonal modulated intraseasonal oscillations in a GCM simulation, Int. J. Climatol., 18, 1521-1537, 1998.

Imbrie, J. and Imbrie, J.: Modeling the climatic response to orbital variations, Science, 207, 943-953, 1980.

Jin, F.-F., Neelin, J., and Ghil, M.: El Niño Southern Oscillation and the annual cycle: Subharmonic frequency-locking and aperiodicity, Physica D, 98, 442-465, 1996.

Klein, P. and Pedlosky, J.: A numerical study of baroclinic instability at large supercriticality, J. Atmos. Sci., 43, 1243-1262, 1986.

Kociuba, G. and Heckenberg, N.: Controlling the complex Lorenz equations by modulation, Phys. Rev. E, 66, art. no. 026205, 2002.

Lathrop, D. and Kostelich, E.: Characterization of an experimental strange attractor by periodic orbits, Phys. Rev. A, 40, 4028-4031, 1989.

Lima, R. and Pettini, M.: Suppression of chaos by resonant parametric perturbations, Phys. Rev. A, 41, 726-733, 1990.

Lorenz, E.: Seasonal and irregular variations of the northern hemisphere sea-level pressure profile, J. Meteorol., 7, 52-59, 1951.

Lorenz, E.: Deterministic nonperiodic flow, J. Atmos. Sci., 20, 130$141,1963$.

Lovegrove, A.: Bifurcations and instabilities in rotating two-layer fluids, $\mathrm{PhD}$ thesis, University of Oxford, 1997.

Lovegrove, A., Moroz, I., and Read, P.: Bifurcations and instabilities in rotating two-layer fluids: I. f-plane, Nonlin. Processes Geophys., 8, 21-36, 2001,

SRef-ID: 1607-7946/npg/2001-8-21.

Lovegrove, A., Moroz, I., and Read, P.: Bifurcations and instabilities in rotating two-layer fluids: II. $\beta$-plane, Nonlin. Processes Geophys., 9, 289-309, 2002,

SRef-ID: 1607-7946/npg/2002-9-289.

Mirius, K. and Sprott, J.: Controlling chaos in low- and highdimensional systems with periodic parametric perturbations, Phys. Rev. E, 59, 5313-5324, 1999.

Namias, J.: The index cycle and its role in the general circulation, J. Meteor., 7, 130-139, 1950.

Nicolis, C.: Self-oscillations and predictability in climate dynamics - periodic forcing and phase locking, Tellus, 36A, 217-227, 1984.

Park, E.-H., Zaks, M., and Kurths, J.: Phase synchronization in the forced Lorenz system, Phys. Rev. E, 60, 6627-6638, 1999.

Pedlosky, J.: Finite-amplitude baroclinic waves, J. Atmos. Sci., 27, 15-30, 1970.

Pedlosky, J.: Finite-amplitude baroclinic waves with small dissipation, J. Atmos. Sci., 28, 587-597, 1971.

Pedlosky, J.: Limit cycles and baroclinic waves, J. Atmos. Sci., 28, 
53-63, 1972.

Pedlosky, J. and Thomson, J.: Baroclinic instability of timedependent currents, J. Fluid. Mech., 490, 189-215, 2003.

Pfeffer, R., Buzyna, G., and Kung, R.: Time-dependent modes of behaviour of thermally driven rotating fluids, J. Atmos. Sci., 37 , 2129-2149, 1980.

Phillips, N.: Energy transformations and meridional circulations associated with simple baroclinic waves in a two-level, quasigeostrophic model, Tellus, 4, 273-286, 1954.

Pikovsky, A., Rosenblum, M., Osipov, G., and Kurths, J.: Phase synchronization of chaotic oscillators by external driving, Physica D, 104, 219-238, 1997.

Pikovsky, A., Rosenblum, M., and Kurths, J.: Synchronization, Cambridge University Press, 2003.

Randel, W. and Stanford, J.: Structure of medium-scale atmospheric waves in the Southern Hemisphere summer, J. Atmos. Sci., 40, 2312-2318, 1983.

Randel, W. and Stanford, J.: An observational study of mediumscale wave dynamics in the Southern Hemisphere summer. Part I: wave structure and energetics, J. Atmos. Sci., 42, 1172-1188, 1985a.

Randel, W. J. and Stanford, J.: The observed life cycle of a baroclinic instability, J. Atmos. Sci., 42, 1364-1373, 1985 b.

Rossby, C.: Relations between variations in the intensity of the zonal circulation of the atmosphere and the displacements of the semipermanent centers of action, J. Mar. Res., 3, 38-55, 1939.
Saltzman, B., Sutera, A., and Hansen, A.: A possible marine mechanism for internally generated long-period climate cycles, J. Atmos. Sci., 39, 2634-2637, 1982.

Sätherblom, H.-E.: Dynamic control of the reversed-field pinch in numerical magnetohydrodynamic simulations, Phys. Plasmas, 4, 174-178, 1997.

Stephenson, D., Pavan, B., and Bojariu, R.: Is the North Atlantic Oscillation a random walk?, Int. J. Climatol., 20, 1-18, 2000.

Vialard, J., Menkes, C., Anderson, D., and Alonso Balmaseda, M.: Sensitivity of Pacific Ocean tropical instability waves to initial conditions, J. Phys. Oceanogr., 33, 105-121, 2003.

Vohra, S., Fabiny, L., and Bucholtz, F.: Suppressed and induced chaos near resonant perturbation of bifurcations, Phys. Rev. Lett., 75, 65-69, 1995.

Wallace, J.: North Atlantic Oscillation/annular mode: Two paradigms - one phenomenon, Q. J. R. Meteorol. Soc., 126, 791805, Symons Memorial Lecture: delivered 19 May 1999, 2000.

Wolf, A., Swift, J., Swinney, H., and Vastano, J.: Determining Lyapunov exponents from a time series, Physica D, 16, 285-317, 1985.

Zaks, M., Park, E.-H., Rosenblum, M., and Kurths, J.: Alternating Locking Ratios in Imperfect Phase Synchronization, Phys. Rev. Lett., 82, 4228-4231, 1999. 